Kyoto University,

Graduate School of Economics

Discussion Paper Series

An Analysis of Simultaneous Company Defaults Using a Shot Noise Process

Masahiko EGAMI and Rusudan KEVKHISHVILI

Discussion Paper No. E-16-001

Graduate School of Economics

Kyoto University

Yoshida-Hommachi, Sakyo-ku

Kyoto City, 606-8501, Japan

April, 2016 


\title{
AN ANALYSIS OF SIMULTANEOUS COMPANY DEFAULTS USING A SHOT NOISE PROCESS
}

\author{
MASAHIKO EGAMI AND RUSUDAN KEVKHISHVILI
}

\begin{abstract}
During subprime mortgage crisis, it became apparent that incumbent models had underestimated company default correlations. Complex models that attempt to incorporate default dependency are difficult to implement in practice. On the contrary, practical models, such as One-Factor Gaussian Copula model, greatly underestimated simultaneous default probabilities. In this article, we develop a model for a company asset process and based on this model, we calculate simultaneous default probabilities using option-theoretic approach. Our model focuses on one industry and includes a shot noise process in the asset model directly. The risk factor driving the shot noise process is common to all companies in the industry but the shot noise parameters are assumed to be company-specific; therefore, every company responds to this common risk factor differently. Apart from the shot noise process, the asset model includes company specific Brownian motion. Compared to commonly used geometric Brownian motion asset model in option-theoretic approach, our model predicted higher simultaneous default probabilities for Citigroup Inc. in 2008, and for all company combinations for the years of 2009 and 2010. Our model is easy to implement and can be extended to analyze any finite number of companies without greatly increasing computational difficulty.
\end{abstract}

Key words: shot noise; option-theoretic approach; asset process; simultaneous default probabilities JEL Classification: G01, G21, G32

\section{Introduction}

This paper aims to calculate simultaneous default probabilities of multiple companies. Our research is motivated by the fact that the incumbent models did not predict the default correlations in the global 
financial crisis; simultaneous default probabilities were underestimated in the structuring and pricing of the CDO's (collateralized debt obligations). We wish to compute default correlations more accurately to enhance risk management quality for the portfolios of debt instruments.

We analyze company defaults through the behavior of its asset process. For this, we define default as an event in which a company's asset value falls below a certain level. It is highly likely that company defaults in one industry are correlated. To incorporate this correlation into the calculation of simultaneous default probabilities, we include a common shot noise process in each company's asset model. In our model, each company's asset value is driven by the company-specific risk factor and by the shot noise process, the latter being common for all companies and having negative effects on the asset value. The shot noise process can be seen as an aggregation of jumps up to each point in time. The effect of jumps does not disappear immediately but decreases gradually over time. This allows us to keep the negative effect of external shocks for a certain period of time. We assume that the parameters of this shot noise process are company-specific. This means that the sensitivity of each company to the jumps of the shot noise process is different. For the computational simplicity, in this paper we will only deal with negative jumps. Our focus is on the subprime mortgage crisis. Therefore, we will be analyzing data from 2004 to 2014 .

Two things are worth noting about our model. First, it tries to capture correlation of company defaults through the shot noise process. We use this shot noise process in an innovative way by including it directly into the asset model. Second, our model is computationally easy to implement. Even though sensitivity to shocks differs from company to company, the driving force of the shot noise process is common. Therefore, by including one shot noise process in the model, we can capture the correlation of any number of companies. As a result, adding one more company to the analysis does not increase computational burden.

In general, there are top-down and bottom-up approaches to default correlation analysis. An example of the top-down approach is Giesecke, Goldberg, and Ding [11]. They propose an intensity-based model for the portfolio loss process and decompose the portfolio intensity into the sum of the intensities of the constituents. They derive the formula for the default probability of the constituent parties as a function of the thinning process and the portfolio intensity. Kunisch and Uhrig-Homburg [15] also take the topdown approach but their method is based on the structural model of a firm as well. They use random thinning to decompose economy's intensity that is driven by macroeconomic factors into the intensities of defaultable company subsets. For this, they define default of a company as an event in which company asset falls at the outstanding debt level. This study employs structural framework similar to Merton [20] and assumes that asset process follows geometric Brownian motion and assets of different companies are correlated. Under these assumptions, they derive solution to the default probability of a certain subset, 
and finally, they calculate thinning probabilities using these default probabilities.

Common shocks are often included in intensity-based models that belong to the bottom-up approach. Examples of these studies are Dong, Wang, and Yuen [4], Herbertsson, Jang, and Schmidt [12], Liang and Wang [18]. This type of studies use shocks to model default intensity processes and derive explicit formulas for the joint probability of default. However, the feasibility of this approach in the analysis of simultaneous defaults of more than 2 companies is not sufficiently explored. Shot noise model is also used in insurance modeling, e.g. Schmidt [24], Jang and Dassios [13]. Another example of the bottom-up approach is a copula model. The Gaussian copula is relatively easy to implement in practice; however, during subprime mortgage crisis it has proved to be insufficient for analyzing multiple defaults since it does not incorporate dependence structure among companies well enough (see e.g. Salmon [23]). Even though there is a wide variety of choices for copula models, to our knowledge, there does not exist a good copula model for multivariate analysis that is not too complex. An example of the copula approach that is based on the asset process is Giesecke [10]. He describes the model where the threshold at which a firm goes bankrupt is not publicly known because the information about firm's liabilities is not fully disclosed. Taking the connection of different firms into account, bond investors estimate threshold levels for firms using available information. Then, while observing the asset dynamics and default events, they update their estimates. This study uses copula to model the dependence structure among thresholds of different companies. Copula of default times is modeled using asset and threshold dependence structures. Another example is Dalla Valle et al. [2]. They employ the pair copula to model dependence structure among current and long-term portions of the asset and debt of the company, and express the company equity as a function of the asset and debt using pair copula. They simulate the values of equity and define default as an event where equity is at or below zero, but they do not describe the extension of the model to simultaneous defaults of multiple companies and its feasibility.

Finally, we give an example of the model that takes completely different approach from the ones mentioned above. This is the study by Filiz et al. [8]. They use graphical model and construct a graph with nodes where each node denotes a firm. They define a binary random variable for each firm that describes default and survival and set up the formula for the joint distribution of these binary random variables. During simulation, they estimate number of defaulted firms in each time interval, and firm nodes are removed from graph with some probability. Under certain homogeneity assumptions, they claim that the number of firm nodes remaining and the total number of defaulted firms follow two-state discrete Markov chain depending only on the previous values. They derive transition probability matrix for this Markov chain and analyze CDO pricing under this framework.

Our model belongs to the bottom-up approach. It is an improvement over the model in Lehar [17] 
that does not allow for dependency between assets of different companies. In contrast to the above mentioned intensity-based models, our model does not use intensity but incorporates shot noise process directly into the asset model. This is the innovative part. Also, in contrast to copula models, our model is computationally easy to implement. In this paper, we present results about three companies. However, this model works for any number of companies and adding a new company to the model does not increase computational difficulty since the shot noise process is assumed to be common for all companies that belong to that particular industry. We estimated simultaneous default probability matrices by our shot noise model and by the model used in Lehar [17]. The important result is that our model predicted higher default probabilities for 2008 (Citigroup Inc.), 2009, and 2010. Also, estimation and simulation was computationally easy.

\section{Methodology}

\section{$2.1 \quad$ Model}

For an asset value process, we want to use a model that incorporates a shot noise process. To make the model simple, we will use only one shot noise process for bad news, which means that the shot noise process will have negative effects on the asset value. The shot noise process at time $t, \lambda_{t}$, is given by the following equation

$$
\lambda_{t}=\lambda_{0} e^{-\delta t}+\sum_{i=1}^{M_{t}} Y_{i} e^{-\delta\left(t-S_{i}\right)}
$$

where $\lambda_{0}$ is the initial value of the shot noise process, $\delta$ is the exponential decay rate, $\left\{S_{i}\right\}_{i=1,2, \ldots}$ are event times of Poisson process $M_{t}$ with constant rate $\rho$, and $\left\{Y_{i}\right\}_{i=1,2, \ldots}$ is a sequence of independent and identically distributed random variables with distribution function $G(y), y>0$. The $Y$ 's represent the size of the shot noise jumps and are independent of $M_{t}$.

This shot noise process is used by Dassios and Jang [3]. The purpose of using this process is that it represents jumps and the effects of jumps do not disappear immediately and remain for a certain period of time. We will also need first and second moments of the jumps which, following Dassios and Jang [3], are assumed to be finite.

$E\left(Y_{i}\right)=\mu_{1}<\infty \quad E\left(Y_{i}^{2}\right)=\mu_{2}<\infty$

Since all jumps are assumed to be positive, we will use $-\lambda_{t}$ to incorporate bad news in the model below. We assume that $\lambda_{0}, \delta$, and $\rho$ differ among companies. We model the asset value process $V$ by the 
combination of geometric Brownian motion and the shot noise process:

$$
V_{t}=e^{X_{0}+\left(\mu-\frac{1}{2} \sigma^{2}\right) t+\sigma B_{t}^{(1)}-\lambda_{t}}
$$

where $V_{t}$ denotes asset value at time t, $\mu$ and $\sigma$ are constant parameters, $B_{t}^{(1)}$ is standard Brownian motion, and $X_{0}$ is some constant. We will use another standard Brownian motion $B_{t}^{(2)}$ in the approximation of the shot noise process. To estimate parameters, we employ the approximation of the shot noise process introduced by Dassios and Jang [3]. For this approximation to hold, we need to assume that the event arrival rate $\rho$ is large enough. This means that events in this model will not be catastrophes, but rather "common events of high frequency" (Dassios \& Jang [3, p. 97]). $\rho$ is the expected number of events that happen during one unit of a time interval. In our estimations, one unit of the time interval is 1 month. In this paper we will focus on one particular industry and $\rho$ can be seen as the number of reported news affecting that industry. Every day a lot of information is reported. Aggregating this for a month means that $\rho$ is quite large; therefore, our assumption is not unnatural.

Following Dassios and Jang [3], we assume that $\lambda_{0}$ is a random variable independent of everything else and has a stationary distribution, such that $\frac{\lambda_{0}-\frac{\mu_{1} \rho}{\delta}}{\sqrt{\frac{\mu_{2} \rho}{2 \delta}}} \stackrel{d}{\rightarrow} Z_{0}$. Let $Z_{t}^{\rho}=\frac{\lambda_{t}-\frac{\mu_{1} \rho}{\delta}}{\sqrt{\frac{\mu_{2} \rho}{2 \delta}}}$. Then from Theorem 2 in Dassios and Jang [3], $Z_{t}^{\rho} \stackrel{d}{\rightarrow} Z_{t}$, where $d Z_{t}=-\delta Z_{t} d t+\sqrt{2 \delta} d B_{t}^{(2)}$.

$B_{t}^{(2)}$ is a standard Brownian motion and we assume $B_{t}^{(1)}$ is independent of $B_{t}^{(2)}$. For simplicity, we also assume that $Z_{0}$ is constant. $Z_{t}$ can equivalently be written as

$$
Z_{t}=Z_{0} e^{-\delta t}+\sqrt{2 \delta} \int_{0}^{t} e^{-\delta(t-s)} d B_{s}^{(2)}
$$

and for $t$ fixed, $Z_{t}$ follows the normal distribution. Hence, we can use $\tilde{\lambda_{t}}=\frac{\mu_{1} \rho}{\delta}+Z_{t} \sqrt{\frac{\mu_{2} \rho}{2 \delta}}$ as Gaussian approximation of $\lambda_{t}$. The asset value process (we will use the same notation $V_{t}$ ) becomes

$$
V_{t}=e^{X_{0}+\left(\mu-\frac{1}{2} \sigma^{2}\right) t+\sigma B_{t}^{(1)}-\frac{\mu_{1} \rho}{\delta}-Z_{t} \sqrt{\frac{\mu_{2} \rho}{2 \delta}}}
$$




\subsection{Dynamics of $V_{t}$ under Approximation}

Since $Z_{t}$ is a semimartingale, the dynamics of the asset process under approximation can be expressed in the following way by Itô's formula (see Appendix A1)

$$
\begin{gathered}
d V_{t}=V_{t}\left(\mu-\frac{1}{2} \sigma^{2}\right) d t+V_{t} \sigma d B_{t}^{(1)}+\frac{1}{2} V_{t} \sigma^{2} d t-V_{t} \sqrt{\frac{\mu_{2} \rho}{2 \delta}} d Z_{t}+\frac{1}{2} V_{t} \frac{\mu_{2} \rho}{2 \delta} d\langle Z\rangle_{t}= \\
=V_{t}\left(\mu+\frac{1}{2} \mu_{2} \rho+\delta \sqrt{\frac{\mu_{2} \rho}{2 \delta}} Z_{t}\right) d t+V_{t} \sigma d B_{t}^{(1)}-V_{t} \sqrt{\mu_{2} \rho} d B_{t}^{(2)}
\end{gathered}
$$

For convenience, let $Q_{t}=\mu+\frac{1}{2} \mu_{2} \rho+\delta \sqrt{\frac{\mu_{2} \rho}{2 \delta}} Z_{t}$. Then, the asset value process follows the differential equation

$$
d V_{t}=V_{t} Q_{t} d t+V_{t}\left(\sigma d B_{t}^{(1)}-\sqrt{\mu_{2} \rho} d B_{t}^{(2)}\right)
$$

We can prove that $V_{t}$ is also a semimartingale (see Appendix A2 for a proof).

\subsection{Risk-neutral Theory}

\subsubsection{One-dimensional Model}

The asset process of the company is not observable in the market. We only know the book value of the assets from the balance sheet. By estimating the unknown parameters (except $\mu_{1}$ and $X_{0}$ because estimation will be conditioned on $\left.V_{0}\right)$ in $(3)$, (4), we will be able to simulate the asset process. For this, first we wish to connect company assets to its equity, since the equity value is observable. We will use the option-theoretic approach to the equity value as in Black and Scholes [1], Merton [20], Lehar [17], and assume that the equity $E_{t}$ is a call option on the company's assets with a strike price equal to future value of the company's debt $D_{T}=D_{t} e^{r(T-t)}$ where $D_{t}$ is the debt of the company at time $t$ and $T$ is the maturity of the option. We need to calculate the value of this call option when the underlying asset follows (5). Lehar [17] assumes that all debt is insured (risk-free) and grows at a risk-free rate $r$. We will also use this assumption for simplicity.

Throughout this paper we will be dealing with a probability space $\left(\Omega, \mathcal{F}_{T}, P\right)$. $T$ can be viewed as any fixed point in time. We assume the filtration is generated by $B_{t}^{(1)}$ and $B_{t}^{(2)}$ and satisfies the usual conditions. Consider the discounted asset value process $e^{-r t} V_{t}$ where $r$ is the constant risk-free rate. 


$$
\begin{aligned}
d\left(e^{-r t} V_{t}\right) & =-r e^{-r t} V_{t} d t+e^{-r t} d V_{t} \\
& =-r e^{-r t} V_{t} d t+e^{-r t} V_{t} Q_{t} d t+e^{-r t} V_{t}\left(\sigma d B_{t}^{(1)}-\sqrt{\mu_{2} \rho} d B_{t}^{(2)}\right) \\
& =e^{-r t} V_{t}\left[\left(-r+Q_{t}\right) d t+\sigma d B_{t}^{(1)}-\sqrt{\mu_{2} \rho} d B_{t}^{(2)}\right]
\end{aligned}
$$

The following argument is based on Shreve [25, pp. 226-228]. Let us define $\alpha_{1}=\sigma$ and $\alpha_{2}=-\sqrt{\mu_{2} \rho}$. Then, we have

$$
d\left(e^{-r t} V_{t}\right)=e^{-r t} V_{t}\left[\left(-r+Q_{t}\right) d t+\sum_{i=1}^{2} \alpha_{i} d B_{t}^{(i)}\right]
$$

In order to make the discounted asset value martingale, we rewrite the equation in the following way:

$$
d\left(e^{-r t} V_{t}\right)=e^{-r t} V_{t} \sum_{i=1}^{2} \alpha_{i}\left[-\theta_{t}^{i} d t+d B_{t}^{(i)}\right]
$$

For an adapted process $\theta_{t}^{i}$ to satisfy the above equation, we need to have

$$
\sum_{i=1}^{2} \alpha_{i} \theta_{t}^{i}=\sigma \theta_{t}^{1}-\sqrt{\mu_{2} \rho} \theta_{t}^{2}=r-Q_{t}
$$

This is one equation in two unknowns; therefore, it has infinitely many solutions. We choose one of them (no matter what we choose, the result will be the same). We let $\theta_{t}^{2}=1$ and $\theta_{t}^{1}=\frac{r-Q_{t}+\sqrt{\mu_{2} \rho}}{\sigma}$. We let $\tilde{B}_{t}^{i}=B_{t}^{i}-\int_{0}^{t} \theta_{s}^{i} d s . \theta_{t}^{1}$ and $\theta_{t}^{2}$ are measurable, adapted processes. If we can show that

$$
H_{t}(\theta)=e^{\sum_{i=1}^{2} \int_{0}^{t} \theta_{s}^{i} d B_{s}^{i}-\frac{1}{2} \int_{0}^{t}\left\|\theta_{s}\right\|^{2} d s}
$$

is a martingale, by Theorem 5.1 (Karatzas \& Shreve [14, p. 191]), $\tilde{B}_{t}$ will be a 2-dimensional standard Brownian motion for $0 \leq t \leq T$ on $\left(\Omega, \mathcal{F}_{T}, \tilde{P}_{T}\right)$ where probability measure $\tilde{P}_{T}$ is defined as $\tilde{P}_{T}(A)=$ $E\left[\mathbb{1}_{A} H_{T}(\theta)\right]$ for $A \in \mathcal{F}_{T}$. Indeed, $H_{T}(\theta)$ is a martingale and we have

$$
d\left(e^{-r t} V_{t}\right)=e^{-r t} V_{t}\left[\sigma d \tilde{B}_{t}^{(1)}-\sqrt{\mu_{2} \rho} d \tilde{B}_{t}^{(2)}\right]=e^{-r t} V_{t} M d \tilde{W}_{t}
$$

as a martingale under $\tilde{P}_{T}$. Here $M=\sqrt{\sigma^{2}+\mu_{2} \rho}$ and $\tilde{W}_{t}=\frac{\sigma}{M} \tilde{B}_{t}^{(1)}-\frac{\sqrt{\mu_{2} \rho}}{M} \tilde{B}_{t}^{(2)}$ is a one-dimensional standard Brownian motion under $\tilde{P}_{T}$ (see Appendix A3).

From (5), we get

$$
\begin{aligned}
d V_{t} & =V_{t} Q_{t} d t+V_{t}\left(\sigma d \tilde{B}_{t}^{(1)}+\left(r-Q_{t}+\sqrt{\mu_{2} \rho}\right) d t\right)-V_{t}\left(\sqrt{\mu_{2} \rho} d \tilde{B}_{t}^{(2)}+\sqrt{\mu_{2} \rho} d t\right) \\
& =V_{t} r d t+V_{t}\left(\sigma d \tilde{B}_{t}^{(1)}-\sqrt{\mu_{2} \rho} d \tilde{B}_{t}^{(2)}\right)=V_{t} r d t+V_{t} M d \tilde{W}_{t}
\end{aligned}
$$


so that

$$
V_{t}=V_{0} e^{\left(r-\frac{1}{2} M^{2}\right) t+M \tilde{W}_{t}}
$$

where $V_{0}=e^{X_{0}-\frac{\mu_{1} \rho}{\delta}-Z_{0} \sqrt{\frac{\mu_{2} \rho}{2 \delta}}}$.

To calculate the price of the above mentioned call option, we will use a risk-neutral pricing formula, i.e. we will take an expectation of the discounted option value at maturity under the risk-neutral measure.

$$
E_{t}=\tilde{E}\left[e^{-r(T-t)}\left(V_{T}-D_{T}\right)^{+}\right]
$$

Here $T$ denotes the maturity of the debt, $E_{t}$ is the equity (i.e., the price of the option written on the value of the firm at time $t$ ) and $D_{T}$ works as the strike price. Observing equation (7), this calculation is the same as in Shreve [25, pp. 219-220] and reduces to famous Black-Scholes formula for the call option price. But in our case, in view of (7), the volatility parameter is $M$. Then, we have,

$E_{t}=V_{t} N\left(d_{t}\right)-D_{t} N\left(d_{t}-M \sqrt{(T-t)}\right)$

where

$d_{t}=\frac{\ln \frac{V_{t}}{D_{t}}+\frac{M^{2}}{2}(T-t)}{M \sqrt{(T-t)}}$

As in Lehar [17], we also assume that $T$ equals 1 year.

\subsubsection{Multidimensional Model}

We can extend our model to any $m$ number of companies. The following argument is again based on Shreve [25, pp. 226-228]. Let's suppose we have a separate $m$ asset value process for each company. $B_{t}^{(i)} i=1,2, \ldots m+1$ is $m+1$-dimensional Brownian motion. We assume the filtration is generated by this Brownian motion. In the similar way as (6), we have

$$
d\left(e^{-r t} V_{t}^{j}\right)=e^{-r t} V_{t}\left[\left(-r+Q_{t}^{j}\right) d t+\sum_{i=1}^{m+1} \alpha_{j, i} d B_{t}^{(i)}\right] \quad j=1,2, \ldots, m
$$

Here $Q_{t}^{j}$ denotes process $Q_{t}$ with parameters of $j$ company. $j$ company's asset value process includes its own Brownian motion $B_{t}^{(j)}$ and shot-noise Brownian motion $B_{t}^{m+1} \cdot \alpha_{j, i}=\sigma^{j}$ if $i=j$. Also, $\alpha_{j, m+1}=$ $\sqrt{\mu_{2}^{j} \rho^{j}}$. Otherwise, this coefficient is zero. $j$ indices indicate that all parameters are company specific. To make the discounted asset values martingale, we again rewrite the equation

$$
d\left(e^{-r t} V_{t}^{j}\right)=e^{-r t} V_{t} \sum_{i=1}^{m+1} \alpha_{j, i}\left[-\theta_{t}^{i} d t+d B_{t}^{(i)}\right]
$$


From this, we get

$$
\sum_{i=1}^{m+1} \alpha_{j, i} \theta_{t}^{i}=r-Q_{t}^{j}
$$

We have $m$ equations in $m+1$ unknowns. This means there are infinitely many solutions. We choose one of them. Again, the result will not depend on our choice.

$$
\begin{gathered}
\alpha_{1,1} \theta_{t}^{1}+\alpha_{1, m+1} \theta_{t}^{m+1}=r-Q_{t}^{1} \\
\alpha_{2,2} \theta_{t}^{2}+\alpha_{2, m+1} \theta_{t}^{m+1}=r-Q_{t}^{2} \\
\cdots \\
\alpha_{m, m} \theta_{t}^{m}+\alpha_{m, m+1} \theta_{t}^{m+1}=r-Q_{t}^{m}
\end{gathered}
$$

We set $\theta_{t}^{m+1}=1$. Then, we get

$$
\theta_{t}^{j}=\frac{r-Q_{t}^{j}-\alpha_{j, m+1}}{\alpha_{j, j}}
$$

In $\forall j Q_{t}^{j}$, the Brownian motion is $B_{t}^{m+1}$. We again are allowed to change the measure (see Appendix A4) and $\tilde{B}_{t}^{(i)}=\int_{0}^{t}-\theta_{s}^{i} d s+B_{t}^{(i)}$ is a Brownian motion under $\tilde{P}_{T}$. We get

$$
d\left(e^{-r t} V_{t}^{j}\right)=e^{-r t} V_{t} \sum_{i=1}^{m+1} \alpha_{j, i} d \tilde{B}_{t}^{(i)}
$$

For each company, we have to estimate the parameters of the asset value process using company equity values. For $j$ company this time we can also combine $\sum_{i=1}^{m+1} \alpha_{j, i} d \tilde{B}_{t}^{(i)}$ as one Brownian motion and use Black-Scholes equation. All calculations will be the same.

\subsection{Estimation Procedure}

To estimate the parameters of $V_{t}$, we use the maximum likelihood estimation technique introduced in Duan [5], Duan [5], and Duan, Gauthier, and Simonato [7]. Given the data of the equity process $E=$ $\left(E_{t}, t=0, \Delta_{t}, 2 \Delta_{t}, \ldots n \Delta_{t}\right)$, we can estimate the parameters by maximizing the following log-likelihood function:

$$
\begin{gathered}
\left.L\left(E_{t}, t=0, \Delta_{t}, 2 \Delta_{t}, \ldots n \Delta_{t}\right) ; \mu, \delta, \mu_{2} \rho, Z_{0}, \sigma\right)= \\
-\frac{n}{2} \ln 2 \pi-\frac{1}{2} \sum_{k=1}^{n} \ln \operatorname{Var}_{k \Delta_{t}}-\sum_{k=1}^{n} \frac{\left(\ln \frac{\hat{V}_{k \Delta_{t}}}{\hat{V}_{(k-1) \Delta_{t}}}-\operatorname{mean}_{k \Delta_{t}}\right)^{2}}{2 \operatorname{Var}_{k \Delta_{t}}}-\sum_{k=1}^{n} \ln \hat{V}_{k \Delta_{t}}-\sum_{k=1}^{n} \ln N\left(\hat{d}_{k \Delta_{t}}\right)
\end{gathered}
$$


where $\hat{V}_{k \Delta_{t}}$ is the unique solution to (8) and $\hat{d}_{k \Delta_{t}}$ is $d_{k \Delta_{t}}$ with $V_{k \Delta_{t}}$ replaced by $\hat{V}_{k \Delta_{t}}$. (see Appendix A5)

$$
\begin{aligned}
\operatorname{mean}_{k \Delta_{t}} & =\left(\mu-\frac{1}{2} \sigma^{2}\right) \Delta_{t}-\sqrt{\frac{\mu_{2} \rho}{2 \delta}} Z_{0} e^{-\delta k \Delta_{t}}\left(1-e^{\delta \Delta_{t}}\right)-\sqrt{\mu_{2} \rho}\left(\frac{1}{e^{\delta \Delta_{t}}}-1\right) e^{-\delta(k-1) \Delta_{t}} \int_{0}^{(k-1) \Delta_{t}} e^{\delta s} d B_{s}^{(2)} \\
\operatorname{Var}_{k \Delta_{t}} & =\sigma^{2} \Delta_{t}+\frac{\mu_{2} \rho}{2 \delta}\left(1-e^{-2 \delta \Delta_{t}}\right)
\end{aligned}
$$

We will be using monthly data; therefore, we set $\Delta_{t}=\frac{1}{12}$. The time to maturity will be assumed as one year, i.e. $T-t=1 \quad \forall t$. The function that we will maximize to get the parameters consists of two parts. Inside the function, $\hat{V}_{k \Delta_{t}}$ will be estimated by fixed-point iteration procedure from (8) using available equity values and after that, using these estimated asset values, the log-likelihood in (9) will be calculated. The result will be a function in the unknown five parameters and this function will be maximized by the built-in interior-point method in Matlab. During this procedure, we will need the values of $\left(\operatorname{mean}_{k \Delta_{t}}\right)$ which involves values of $e^{-\delta(k-1) \Delta_{t}} \int_{0}^{(k-1) \Delta_{t}} e^{\delta s} d B_{s}^{(2)}$. This process is not directly observable. Therefore, we will simulate this process and use the simulated values in the calculation of the log-likelihood. For future research, this way of estimation can be improved if we somehow infer the values of this unobservable process from the values of $\hat{V}_{t}$. Also, if we employ an estimating procedure during which we can use estimated new parameters at next step, updating the values of this process can also improve the estimation.

\section{Data}

The data used in the estimations is obtained from Thomson Reuters Datastream and Worldscope and is displayed in millions of units of local currency. Our focus is on the banking industry; however, the above mentioned model can be used in the analysis of any industry. The choice of companies can be random. We will be analyzing three companies: JPMorgan Chase \& Co. (JPM), Citigroup Inc. (Citi), and Bank of America Corporation (BAC) (see the company profiles). Market Value by Company (MVC) from Datastream will be used as the equity data. Market Value (MV) from Datastream is the market value of only one class of shares. MVC is the same as MV for the companies with only one listed equity security. However, for the companies with more than one type of listed shares or unlisted shares, MVC takes these other types of shares into consideration too. For the detailed description, refer to Datastream Datatype Definition. For the estimation, we will be using monthly data from 2004/12/30 to 2014/12/30, i.e. the values of the 28 th or 29 th days from February and the 30th day from the other months (121 
observations in total). Graph 1 displays the monthly MVC for the three companies.

Table 1: JPM Company Profile

\begin{tabular}{cccccccc} 
Year & 2004 & 2005 & 2006 & 2007 & 2008 & 2009 & 2010 \\
\hline \hline Total Assets & 1157248 & 1198942 & 1351520 & 1562147 & 2175052 & 2031989 & 2117605 \\
Total Liabilities & 1051595 & 1091731 & 1235730 & 1438926 & 2008168 & 1866624 & 1941499 \\
ROE & $5.87 \%$ & $7.98 \%$ & $12.96 \%$ & $12.86 \%$ & $3.82 \%$ & $6.01 \%$ & $9.69 \%$ \\
ROA & $0.63 \%$ & $0.95 \%$ & $1.67 \%$ & $1.35 \%$ & $0.59 \%$ & $0.75 \%$ & $0.96 \%$ \\
Net Income & $-33.53 \%$ & $89.95 \%$ & $70.27 \%$ & $6.38 \%$ & $-63.52 \%$ & $109.24 \%$ & $39.89 \%$
\end{tabular}

\begin{tabular}{ccccc} 
Year & 2011 & 2012 & 2013 & 2014 \\
\hline \hline Total Assets & 2265792 & 2359141 & 2415689 & 2572773 \\
Total Liabilities & 2082219 & 2155072 & 2204511 & 2341046 \\
ROE & $10.21 \%$ & $10.72 \%$ & $8.40 \%$ & $9.75 \%$ \\
ROA & $1.01 \%$ & $1.06 \%$ & $0.92 \%$ & $1.00 \%$ \\
Net Income & $10.92 \%$ & $12.82 \%$ & $-15.26 \%$ & $21.96 \%$
\end{tabular}

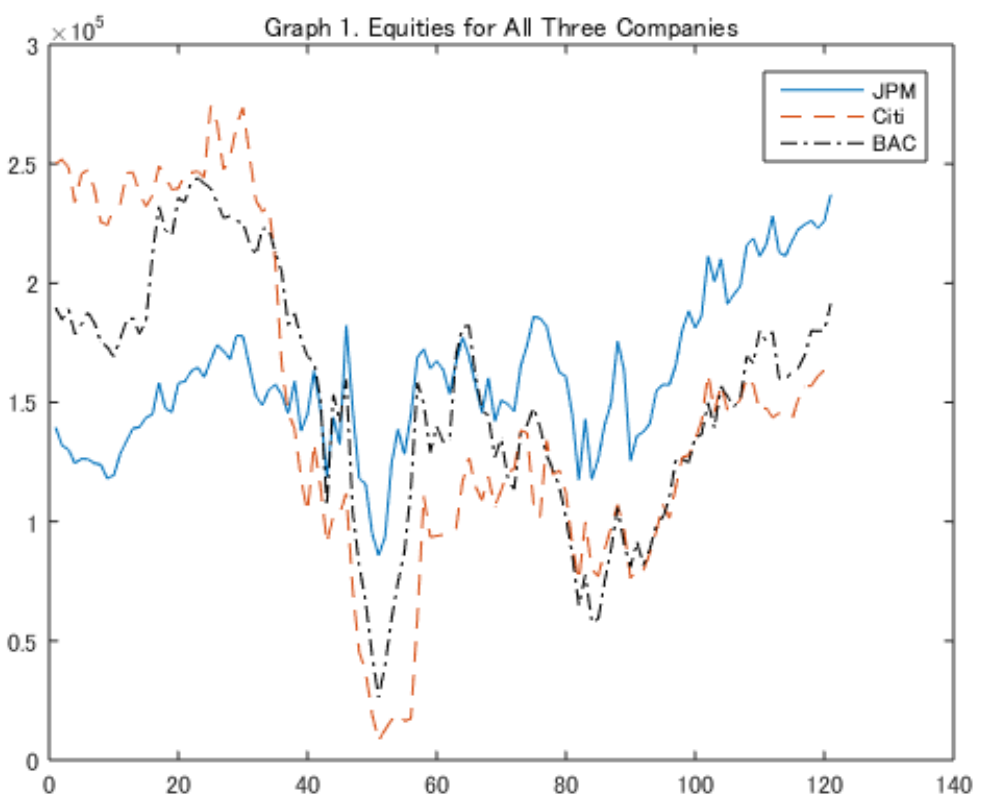

From the company balance sheets, we calculated $D_{t}$ for each $t$. We define $D_{t}$ as the sum of Deposits- 
Table 2: Citi Company Profile

\begin{tabular}{cccccccc} 
Year & 2004 & 2005 & 2006 & 2007 & 2008 & 2009 & 2010 \\
\hline \hline Total Assets & 1484101 & 1494037 & 1884318 & 2187631 & 1938470 & 1856646 & 1913902 \\
Total Liabilities & 1374810 & 1381500 & 1764535 & 2074033 & 1794448 & 1701673 & 1748113 \\
ROE & $16.56 \%$ & $22.33 \%$ & $18.66 \%$ & $3.08 \%$ & $-31.88 \%$ & $-8.28 \%$ & $6.66 \%$ \\
ROA & $1.49 \%$ & $2.00 \%$ & $1.73 \%$ & $0.72 \%$ & $-0.84 \%$ & $0.10 \%$ & $0.98 \%$ \\
Net Income & $-4.52 \%$ & $44.25 \%$ & $-12.41 \%$ & $-83.21 \%$ & - & - & - \\
& & & & & & & \\
& Year & 2011 & 2012 & 2013 & 2014 & \\
\cline { 2 - 7 } & Total Assets & 1818846 & 1864660 & 1880382 & 1842530 & \\
& Total Liabilities & 1639273 & 1673663 & 1674249 & 1630485 & \\
ROE & $6.37 \%$ & $4.04 \%$ & $6.88 \%$ & $3.37 \%$ & \\
ROA & - & $0.72 \%$ & - & - & \\
Net Income & $3.51 \%$ & $-32.22 \%$ & $81.83 \%$ & $-46.29 \%$ &
\end{tabular}

Table 3: BAC Company Profile

\begin{tabular}{cccccccc} 
Year & 2004 & 2005 & 2006 & 2007 & 2008 & 2009 & 2010 \\
\hline \hline Total Assets & 1110432 & 1291803 & 1459737 & 1715746 & 1817943 & 2230232 & 2264909 \\
Total Liabilities & 1010197 & 1190270 & 1324465 & 1568943 & 1640891 & 1998788 & 2036661 \\
ROE & $19.18 \%$ & $16.35 \%$ & $18.07 \%$ & $10.77 \%$ & $1.82 \%$ & $-1.33 \%$ & $-1.77 \%$ \\
ROA & $1.70 \%$ & $1.61 \%$ & $1.87 \%$ & $1.33 \%$ & $0.59 \%$ & $0.81 \%$ & $0.30 \%$ \\
Net Income & $30.83 \%$ & $18.05 \%$ & $28.35 \%$ & $-29.11 \%$ & $-73.25 \%$ & $59.18 \%$ & - \\
& & & & & & & \\
& Year & 2011 & 2012 & 2013 & 2014 & \\
\cline { 3 - 8 } & Total Assets & 2097047 & 2176936 & 2102273 & 2104534 & \\
& Total Liabilities & 1866946 & 1939980 & 1869588 & 1861063 & \\
& ROE & $0.04 \%$ & $1.29 \%$ & $4.61 \%$ & $1.71 \%$ & \\
ROA & $0.42 \%$ & $0.48 \%$ & - & - & \\
& Net Income & - & $189.69 \%$ & $173.03 \%$ & $-57.71 \%$ &
\end{tabular}


Total, Commercial paper, Debt and equity instruments-Trading liabilities, Federal funds purchased under repurchase agreement, other borrowed funds, and one half of Long Term Debt. This addition of one half of the long term debt is a conventional method adopted by Moody's KMV, a unit that offers commercial packages of default probability. For JPM, we have all these categories available (Federal funds purchased under repurchase agreements is displayed under the name of under repurchase agreements). However, for the other two companies the data is not available under these names and we make small adjustments.

For Citi, we define $D_{t}$ as the sum of Deposits-Total, Federal funds purchased and securities (sum of Federal Funds Purchased, Security Sold under Repurchase Agreement, and Federal funds purchased and securities), Commercial paper, Trading account liabilities, Short-term borrowings (sum of Short-term borrowings and Other borrowings - Balancing value), and one half of Long-term debt. For BAC, $D_{t}$ is the sum of Deposits-Total, Federal funds purchased and securities (sum of Federal Funds Purchased, Security Sold under Repurchase Agreement, and Federal funds purchased and securities), Trading account liabilities, short term borrowings, and one half of Long term debt. Commercial paper data was not available on the balance sheet for this company.

Deposits-Total for all companies and Long term debt for BAC are retrieved from Worldscope. The values are restated, updated or reclassified values, i.e. the latest values available. The rest of the data is from Reuters. This debt data is quarterly. We used interpolation to obtain the values of the debt for the missing months. Graph 2 displays the time-series (121 observations) of the debt for all companies.

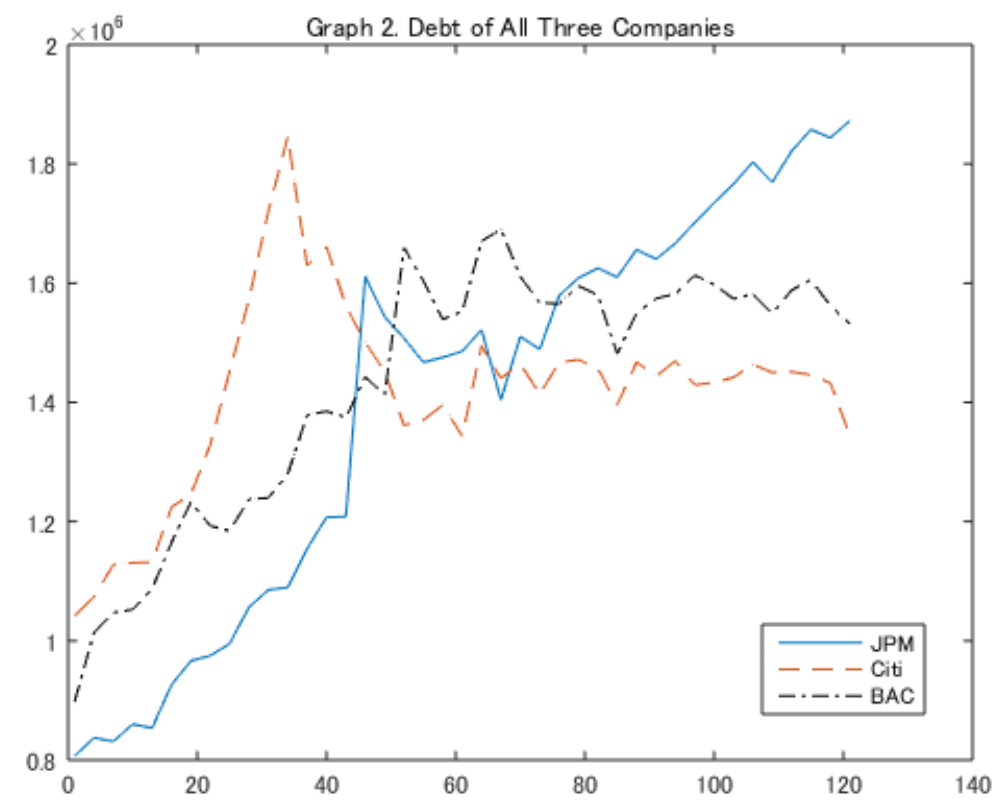


The equity data is also available on the weekly basis. However, the most detailed debt data is quarterly. If we try to retrieve the missing weekly points for debt, the missing points for the interpolation will be too many. We think that if we use the weekly data, there will be a lot of uncertainties about the correctness of the interpolated debt levels. In the case of the monthly data, we have greater confidence that the equity and the debt values match each other at each point in time.

\section{Estimation, Results, and Comparison}

Since there is a possibility that parameters of the model change by time, we split the estimation period into 2-year subperiods: 2004/12/30 2006/12/30, 2005/12/30 2007/12/30, 2006/12/30 2008/12/30,

2007/12/30 2009/12/30, 2008/12/30 2010/12/30, 2009/12/30 2011/12/30, 2010/12/30 2012/12/30, 2011/12/30 2013/12/30, 2012/12/30 2014/12/30. For each 2-year interval, we estimated the parameters of our model and of the model used in Lehar [17]. We will call the latter one GBM model, since it assumes that the asset value follows geometric Brownian motion. Thus, the difference between these two models is that the first one incorporates the shot noise process and the second one does not. For comparison, we report the parameter values of the two models together for each period.

Using the estimated parameters of the 2-year subinterval, we generated the asset value process for the next year. For this, first we retrieved starting value of the asset process from the Black-Scholes equation using the estimated variance parameter and starting from that value, we simulated the next 12 observations. For example, if we used data from 2004/12/30 2006/12/30 to estimate parameters, we retrieved the starting asset value using the Black-Scholes formula (8), variance, and the equity of 2006/12/30. Finally, we compared the last simulated asset value to the debt of the company. We assume that debt grows at the rate of 1-year treasury bill $r$, i.e. for comparison with assets, in our example, we used the year-end debt level of 2006 multiplied by $e^{r}$, where $\mathrm{r}$ is the last observed treasury rate in the year of 2006. Table 12 in Appendix B summarizes the interest rates used in the calculation of the debt. If the asset value fell at or below the level of debt $D_{T}$, we considered this as default. We simulated the paths of the asset value process 10,000 times and counted the number of defaults.

Because we also use simulation to calculate the likelihood function value, the maximum likelihood estimation of our model does not give only one set of parameter solution. Therefore, we did the maximization many times, i.e. under many simulated data. First, we chose those parameter vectors that do not have $\sigma$ very small compared to the one estimated in GBM model. Using these estimated parameter vectors, we simulated the asset value process for the same 2-year period that we used in the approxima- 
tion and backed out the equity values from the Black-Scholes equation (8). Then, we compared these equity values to the actual equity of the company. We chose the parameters that gave relatively good approximation of the equity process. We also display the standard errors (in parenthesis) calculated by inverting the Hessian matrix of the constrained minimization problem and taking the square root of the diagonal elements; however, from the description of Matlab manual of the function fmincon, the reported standard errors may not be accurate. We have also observed that the standard errors depend on the initial values. In tables that report parameter values, "Year" indicates the year for which we did the simulation. This means that the parameters are obtained from the previous two years. "Default" shows the probability of defaulting at the end of the indicated year. The graphs that compare the calculated equity values and real equity values are displayed in Appendix B. 


\subsection{Results (1) 2007-2010}

Table 4: Simultaneous Default Matrices

\begin{tabular}{|c|c|c|c|}
\hline \multicolumn{4}{|c|}{ GBM Model } \\
\hline 2007 & JPM & Citi & BAC \\
\hline JPM & 0 & 0 & 0 \\
\hline Citi & 0 & 0 & 0 \\
\hline BAC & 0 & 0 & 0 \\
\hline All & \multicolumn{3}{|c}{0} \\
\hline
\end{tabular}

\begin{tabular}{|c|c|c|c|}
\hline \multicolumn{4}{|c|}{ Shot Noise Model } \\
\hline 2007 & JPM & Citi & BAC \\
\hline JPM & 0 & 0 & 0 \\
\hline Citi & 0 & 0 & 0 \\
\hline BAC & 0 & 0 & 0 \\
\hline All & \multicolumn{3}{|c|}{0} \\
\hline
\end{tabular}

\begin{tabular}{|c|c|c|c|}
\hline \multicolumn{4}{|c|}{ GBM Model } \\
\hline 2008 & JPM & Citi & BAC \\
\hline JPM & 0 & 0 & 0 \\
\hline Citi & 0 & 0.0528 & 0 \\
\hline BAC & 0 & 0 & 0 \\
\hline All & \multicolumn{3}{|c}{0} \\
\hline
\end{tabular}

\begin{tabular}{|c|c|c|c|}
\hline \multicolumn{4}{|c|}{ Shot Noise Model } \\
\hline 2008 & JPM & Citi & BAC \\
\hline JPM & 0 & 0 & 0 \\
\hline Citi & 0 & 0.0609 & 0 \\
\hline BAC & 0 & 0 & 0 \\
\hline All & \multicolumn{3}{|c}{0} \\
\hline
\end{tabular}

\begin{tabular}{|c|c|c|c|}
\hline \multicolumn{4}{|c|}{ GBM Model } \\
\hline 2009 & JPM & Citi & BAC \\
\hline JPM & 0.1925 & 0.1741 & 0.0489 \\
\hline Citi & 0.1741 & 0.9167 & 0.2413 \\
\hline BAC & 0.0489 & 0.2413 & 0.2565 \\
\hline All & \multicolumn{3}{|c}{0.0478} \\
\hline
\end{tabular}

\begin{tabular}{|c|c|c|c|}
\hline \multicolumn{4}{|c|}{ Shot Noise Model } \\
\hline 2009 & JPM & Citi & BAC \\
\hline JPM & 0.3411 & 0.3086 & 0.1049 \\
\hline Citi & 0.3086 & 0.9194 & 0.2655 \\
\hline BAC & 0.1049 & 0.2655 & 0.2788 \\
\hline All & \multicolumn{3}{|c}{0.0948} \\
\hline
\end{tabular}

\begin{tabular}{|c|c|c|c|}
\hline \multicolumn{4}{|c|}{ GBM Model } \\
\hline 2010 & JPM & Citi & BAC \\
\hline JPM & 0.2328 & 0.1639 & 0.0487 \\
\hline Citi & 0.1639 & 0.7399 & 0.1521 \\
\hline BAC & 0.0487 & 0.1521 & 0.2132 \\
\hline All & \multicolumn{3}{|c}{0.0379} \\
\hline
\end{tabular}

\begin{tabular}{|c|c|c|c|}
\hline \multicolumn{4}{|c|}{ Shot Noise Model } \\
\hline 2010 & JPM & Citi & BAC \\
\hline JPM & 0.3312 & 0.2992 & 0.0885 \\
\hline Citi & 0.2992 & 0.8882 & 0.2216 \\
\hline BAC & 0.0885 & 0.2216 & 0.2478 \\
\hline All & \multicolumn{3}{|c}{0.0804} \\
\hline
\end{tabular}


Refer to Table 4 on the previous page. "All" indicates the probability that all of the three banks default within one year. In the years of 2009 and 2010, the probabilities predicted by the shot noise process are twice as large as those by the GBM. We can confirm this feature by also comparing the pair-wise default probabilities. As of the goodness of fit, we review the graphs in Appendix B. In each graph, we plot, by the dashed lines, the actual equity value, and, by the solid lines, the implied equity value. The latter is retrieved from the asset value estimated by the shot noise model (upper panel) and the GBM (lower panel). In the years of 2005-2006, we may say that the GBM and the shot noise model have similar fits for JPM and Citi. In case of BAC, the shot noise model displays better fit. In 2006-2007, the shot noise model has better fit for JPM and Citi. In case of BAC, we may say that the GBM model has slightly better fit.

We should note that in the year of 2008, Citi has positive default probability in both models. The corresponding equity values (based on which the default probabilities for the year 2008 are computed) are shown in Graph 8 in Appendix B. In the case of the shot noise process, while we have reported the parameters that provide the best fit, we encountered certain parameter sets that lead to higher default probabilities for the year 2008. From Graph 8, we can see that towards the end the retrieved equity has upward trend in both models. We obtained a parameter vector with small $\sigma$ (around 0.01 times the GBM $\sigma$ ) based on which the retrieved equity does not go upward in the end, and the gap between actual and retrieved equities is maintained. For this parameter vector, the default probability was around 14\%. A possible explanation is that although Citi's equity price was not performing badly in the year of 2006-2007, its asset quality has deteriorated but was not necessarily reflected in the market price of equity. But if the shot noise process were to be used during that period, that trend could have been detected. Note that Citi was bailed out in 2008 (see Wilchins and Stempel [27]). Compared to the model in Lehar [17], our model does predict some kind of threat of the subprime crisis for Citi. Hence, in the near future, we wish to enhance the accuracy of parameter estimation of the shot noise process so that we could have more insight in what was really happening in the years of 2006-2007.

For the year of 2009, the shot noise model has better fit for JPM (Graph 5) and BAC (Graph 13). In case of Citi, $\sigma$ for the shot noise model has a tendency to be much smaller than the GBM $\sigma$ (around 100 times smaller). We obtained only one parameter vector where $\sigma$ was not very small, and we report this solution (see Graph 9). Other parameter vectors where $\sigma$ was very small gave different default probabilities, some near 1 and some lower than the GBM model. For the year of 2010, again, the shot noise process seems to give better fit (with higher joint probabilities). See Graphs 6, 10, and 14 for JPM, Citi, and BAC, respectively. 
Table 5: Model Parameters for JPM

\begin{tabular}{|c|c|c|c|c|c|c|c|c|}
\hline Year & \multicolumn{4}{|c|}{2007} & \multicolumn{4}{c|}{2008} \\
\hline Model & \multicolumn{2}{|c|}{ GBM } & \multicolumn{2}{|c|}{ Shot Noise } & \multicolumn{2}{c|}{ GBM } & \multicolumn{3}{c|}{ Shot Noise } \\
\hline$\mu$ & 0.1035 & $(0.0143)$ & 0.1087 & $(0.0209)$ & 0.1354 & $(0.0238)$ & 0.0936 & $(0.0284)$ \\
\hline$\sigma$ & 0.0295 & $(0.0039)$ & 0.0288 & $(0.0042)$ & 0.0324 & $(0.0044)$ & 0.0262 & $(0.0042)$ \\
\hline$\mu_{2} \rho$ & & & 0.0000 & $(0.0000)$ & & & 0.0000 & $(0.0001)$ \\
\hline$Z_{0}$ & & & -11.2869 & $(21.8202)$ & & & 48.2436 & $(163.0164)$ \\
\hline$\delta$ & & & 20.0351 & $(3.3368)$ & & & 3.8692 & $(1.8205)$ \\
\hline$M$ & & & 0.0291 & & & & 0.0266 & \\
\hline Likelihood & -252.0745 & & -251.5888 & & -257.3866 & & -252.5007 & \\
\hline Default & 0.0000 & & 0.0000 & & 0.0000 & & 0.0000 & \\
\hline
\end{tabular}

\begin{tabular}{|c|c|c|c|c|c|c|c|c|}
\hline Year & \multicolumn{4}{|c|}{2009} & \multicolumn{4}{c|}{2010} \\
\hline Model & \multicolumn{2}{|c|}{ GBM } & \multicolumn{2}{|c|}{ Shot Noise } & \multicolumn{2}{c|}{ GBM } & \multicolumn{2}{c|}{ Shot Noise } \\
\hline$\mu$ & 0.1719 & $(0.1208)$ & 0.0852 & $(0.1052)$ & 0.1361 & $(0.1510)$ & 0.0817 & $(0.1370)$ \\
\hline$\sigma$ & 0.1815 & $(0.3763)$ & 0.1450 & $(0.0511)$ & 0.2075 & $(0.0539)$ & 0.1755 & $(0.0526)$ \\
\hline$\mu_{2} \rho$ & & & 0.0225 & $(0.0371)$ & & & 0.0378 & $(0.0559)$ \\
\hline$Z_{0}$ & & & 0.1396 & $(0.5550)$ & & & 0.3237 & $(0.4052)$ \\
\hline$\delta$ & & & 6.4869 & $(1.0552)$ & & & 17.6783 & $(2.5744)$ \\
\hline$M$ & & & 0.2086 & & & & 0.2619 & \\
\hline Likelihood & -287.9526 & & -287.6399 & & -288.4642 & & -287.0984 & \\
\hline Default & 0.1925 & & 0.3411 & & 0.2328 & & 0.3312 & \\
\hline
\end{tabular}


Table 6: Model Parameters for Citi

\begin{tabular}{|c|c|c|c|c|c|c|c|c|}
\hline Year & \multicolumn{4}{|c|}{2007} & \multicolumn{4}{c|}{2008} \\
\hline Model & \multicolumn{2}{|c|}{ GBM } & \multicolumn{2}{c|}{ Shot Noise } & \multicolumn{2}{c|}{ GBM } & \multicolumn{2}{c|}{ Shot Noise } \\
\hline$\mu$ & 0.1457 & $(0.0283)$ & 0.1483 & $(0.0333)$ & 0.1255 & $(0.0716)$ & 0.0895 & $(0.1032)$ \\
\hline$\sigma$ & 0.0399 & $(0.0058)$ & 0.0393 & $(0.0057)$ & 0.0994 & $(0.0192)$ & 0.0940 & $(0.0197)$ \\
\hline$\mu_{2} \rho$ & & & 0.0000 & $(0.0001)$ & & & 0.0015 & $(0.0097)$ \\
\hline$Z_{0}$ & & & -6.4542 & $(28.2323)$ & & & 2.5004 & $(1.0706)$ \\
\hline$\delta$ & & & 18.2629 & $(10.1960)$ & & & 7.8986 & $(1.0091)$ \\
\hline$M$ & & & 0.0397 & & & & 0.1019 & \\
\hline Likelihood & -267.1499 & & -266.8642 & & -289.3226 & & -289.0141 & \\
\hline Default & 0.0000 & & 0.0000 & & 0.0528 & & 0.0609 & \\
\hline
\end{tabular}

\begin{tabular}{|c|c|c|c|c|c|c|c|c|}
\hline Year & \multicolumn{4}{|c|}{2009} & \multicolumn{4}{c|}{2010} \\
\hline Model & \multicolumn{2}{|c|}{ GBM } & \multicolumn{2}{c|}{ Shot Noise } & \multicolumn{2}{c|}{ GBM } & \multicolumn{2}{c|}{ Shot Noise } \\
\hline$\mu$ & -0.1069 & $(0.0908)$ & -0.1068 & $(0.2684)$ & -0.0979 & $(0.1226)$ & -0.1895 & $(0.2059)$ \\
\hline$\sigma$ & 0.1301 & $(0.0367)$ & 0.1299 & $(0.0349)$ & 0.1723 & $(0.0837)$ & 0.1437 & $(0.1277)$ \\
\hline$\mu_{2} \rho$ & & & 0.0001 & $(0.0001)$ & & & 0.0175 & $(0.0611)$ \\
\hline$Z_{0}$ & & & -17.8060 & $(13.1695)$ & & & 1.2752 & $(3.1261)$ \\
\hline$\delta$ & & & 0.0000 & $(0.0097)$ & & & 10.4252 & $(9.7605)$ \\
\hline$M$ & & & 0.1301 & & & & 0.1952 & \\
\hline Likelihood & -286.2191 & & -283.9503 & & -274.5238 & & -272.7072 & \\
\hline Default & 0.9167 & & 0.9194 & & 0.7399 & & 0.8882 & \\
\hline
\end{tabular}


Table 7: Model Parameters for BAC

\begin{tabular}{|c|c|c|c|c|c|c|c|c|}
\hline Year & \multicolumn{4}{|c|}{2007} & \multicolumn{4}{c|}{2008} \\
\hline Model & \multicolumn{2}{|c|}{ GBM } & \multicolumn{2}{|c|}{ Shot Noise } & \multicolumn{2}{c|}{ GBM } & \multicolumn{3}{c|}{ Shot Noise } \\
\hline$\mu$ & 0.1360 & $(0.0248)$ & 0.1052 & $(0.0383)$ & 0.1029 & $(0.0205)$ & 0.0556 & $(0.0335)$ \\
\hline$\sigma$ & 0.0480 & $(0.0076)$ & 0.0411 & $(0.0065)$ & 0.0403 & $(0.0058)$ & 0.0323 & $(0.0094)$ \\
\hline$\mu_{2} \rho$ & & & 0.0000 & $(0.0002)$ & & & 0.0001 & $(0.0007)$ \\
\hline$Z_{0}$ & & & 37.4186 & $(87.3341)$ & & & 22.4712 & $(60.4543)$ \\
\hline$\delta$ & & & 5.9174 & $(3.6974)$ & & & 3.4835 & $(1.3856)$ \\
\hline$M$ & & & 0.0417 & & & & 0.0342 & \\
\hline Likelihood & -269.3417 & & -265.8649 & & -267.5702 & & -263.3168 & \\
\hline Default & 0.0000 & & 0.0000 & & 0.0000 & & 0.0000 & \\
\hline
\end{tabular}

\begin{tabular}{|c|c|c|c|c|c|c|c|c|}
\hline Year & \multicolumn{3}{|c|}{2009} & \multicolumn{4}{c|}{2010} \\
\hline Model & \multicolumn{2}{|c|}{ GBM } & \multicolumn{2}{|c|}{ Shot Noise } & \multicolumn{2}{c|}{ GBM } & \multicolumn{2}{c|}{ Shot Noise } \\
\hline$\mu$ & 0.0146 & $(0.0466)$ & -0.0125 & $(0.0665)$ & 0.0392 & $(0.0788)$ & 0.0092 & 0.0507 \\
\hline$\sigma$ & 0.0660 & $(0.0098)$ & 0.0632 & $(0.0121)$ & 0.1146 & $(0.1432)$ & 0.0988 & 0.0238 \\
\hline$\mu_{2} \rho$ & & & 0.0004 & $(0.0016)$ & & & 0.0014 & 0.0028 \\
\hline$Z_{0}$ & & & 4.2728 & $(8.5715)$ & & & 0.5765 & 0.4628 \\
\hline$\delta$ & & & 7.8314 & $(7.7353)$ & & & 21.1645 & 3.8815 \\
\hline$M$ & & & 0.0664 & & & & 0.1058 & \\
\hline Likelihood & -279.4183 & & -279.0548 & & -280.4275 & & -278.4803 & \\
\hline Default & 0.2565 & & 0.2788 & & 0.2132 & & 0.2478 & \\
\hline
\end{tabular}




\subsection{Results (2) 2011-2014}

Table 8: Simultaneous Default Matrices

\begin{tabular}{|c|c|c|c|}
\hline \multicolumn{4}{|c|}{ GBM Model } \\
\hline 2011 & JPM & Citi & BAC \\
\hline JPM & 0.0579 & 0.0148 & 0.0049 \\
\hline Citi & 0.0148 & 0.2473 & 0.0159 \\
\hline BAC & 0.0049 & 0.0159 & 0.0684 \\
\hline All & \multicolumn{3}{|c|}{0.0012} \\
\hline
\end{tabular}

\begin{tabular}{|c|c|c|c|}
\hline \multicolumn{4}{|c|}{ Shot Noise Model } \\
\hline 2011 & JPM & Citi & BAC \\
\hline JPM & 0.0979 & 0.0425 & 0 \\
\hline Citi & 0.0425 & 0.4233 & 0.0001 \\
\hline BAC & 0 & 0.0001 & 0.0004 \\
\hline All & \multicolumn{3}{|c}{0} \\
\hline
\end{tabular}

\begin{tabular}{|c|c|c|c|}
\hline \multicolumn{4}{|c|}{ GBM Model } \\
\hline 2012 & JPM & Citi & BAC \\
\hline JPM & 0.0898 & 0.0274 & 0.0567 \\
\hline Citi & 0.0274 & 0.2851 & 0.2001 \\
\hline BAC & 0.0567 & 0.2001 & 0.6767 \\
\hline All & \multicolumn{3}{|c|}{0.0159} \\
\hline
\end{tabular}

\begin{tabular}{|c|c|c|c|}
\hline \multicolumn{4}{|c|}{ Shot Noise Model } \\
\hline 2012 & JPM & Citi & BAC \\
\hline JPM & 0.0912 & 0.0009 & 0.0130 \\
\hline Citi & 0.0009 & 0.0038 & 0.0007 \\
\hline BAC & 0.0130 & 0.0007 & 0.1249 \\
\hline All & \multicolumn{3}{|c|}{0.0003} \\
\hline
\end{tabular}

\begin{tabular}{|c|c|c|c|}
\hline \multicolumn{4}{|c|}{ GBM Model } \\
\hline 2013 & JPM & Citi & BAC \\
\hline JPM & 0.0003 & 0.0001 & 0 \\
\hline Citi & 0.0001 & 0.1188 & 0.0115 \\
\hline BAC & 0 & 0.0115 & 0.0987 \\
\hline All & \multicolumn{3}{|c}{0} \\
\hline
\end{tabular}

\begin{tabular}{|c|c|c|c|}
\hline \multicolumn{4}{|c|}{ Shot Noise Model } \\
\hline 2013 & JPM & Citi & BAC \\
\hline JPM & 0 & 0 & 0 \\
\hline Citi & 0 & 0.1066 & 0.0095 \\
\hline BAC & 0 & 0.0095 & 0.0745 \\
\hline All & \multicolumn{3}{|c}{0} \\
\hline
\end{tabular}

\begin{tabular}{|c|c|c|c|}
\hline \multicolumn{4}{|c|}{ GBM Model } \\
\hline 2014 & JPM & Citi & BAC \\
\hline JPM & 0 & 0 & 0 \\
\hline Citi & 0 & 0.0001 & 0 \\
\hline BAC & 0 & 0 & 0 \\
\hline All & \multicolumn{3}{|c}{0} \\
\hline
\end{tabular}

\begin{tabular}{|c|c|c|c|}
\hline \multicolumn{4}{|c|}{ Shot Noise Model } \\
\hline 2014 & JPM & Citi & BAC \\
\hline JPM & 0 & 0 & 0 \\
\hline Citi & 0 & 0 & \\
\hline BAC & 0 & 0 & 0 \\
\hline All & \multicolumn{3}{|c}{0} \\
\hline
\end{tabular}


For the year of 2011, the default probabilities are lower than the previous year. The shot noise model gives better fit and higher default probabilities than the GBM for JPM and Citi (see Graphs 15 and 19). For BAC, the shot noise models gave lower default probability. From Graph 23, we may say that, in case of BAC, the shot noise model catches upward trend in equity better than GBM for the years of 2009-2010. We want to point out that during this time period, the actual equity of Citi and BAC drop to a very low level and then start to increase from that point. We have already mentioned Citi's bailout. BAC was also subject to government bailout in early January of 2009 (see Rucker and Stempel [22]). Therefore, we can say that the increase in the equities of these two companies is not a natural phenomenon, but rather a result of a planned intervention.

For the year of 2012, from both models we get much higher default probability for BAC than in the previous year. This could be linked to the financial crisis in Europe. Freed [9] discusses European crisis and the threats that are imposed on BAC for 2012. The shot noise model gives better fit than the GBM (see Graph 24) and lower default probability. In case of Citi, we obtain lower default probability from the shot noise model than from the GBM. From Graph 20, we can say that the shot noise model catches the equity trend better. For JPM, the shot noise model gives slightly better fit (see Graph 16) and slightly higher default probability.

The probability of all three companies defaulting simultaneously goes to 0 in 2013 . In 2014 , default probabilities for all company combinations go to 0 . We also did the simulation for 2015 . The results were not different from 2014. 
Table 9: Model Parameters for JPM

\begin{tabular}{|c|c|c|c|c|c|c|c|c|}
\hline Year & \multicolumn{4}{|c|}{2011} & \multicolumn{4}{c|}{2012} \\
\hline Model & \multicolumn{2}{|c|}{ GBM } & \multicolumn{2}{|c|}{ Shot Noise } & \multicolumn{2}{c|}{ GBM } & \multicolumn{3}{c|}{ Shot Noise } \\
\hline$\mu$ & 0.0023 & $(0.1464)$ & 0.0155 & $(0.0421)$ & 0.0264 & $(0.0490)$ & 0.0141 & $(1.9828)$ \\
\hline$\sigma$ & 0.0651 & $(0.0112)$ & 0.0611 & $(0.0130)$ & 0.0684 & $(0.0130)$ & 0.0635 & $(0.5683)$ \\
\hline$\mu_{2} \rho$ & & & 0.0001 & $(0.0006)$ & & & 0.0004 & $(0.0007)$ \\
\hline$Z_{0}$ & & & -17.4028 & $(38.4435)$ & & & 1.4197 & $(6.0380)$ \\
\hline$\delta$ & & & 15.7057 & $(10.2284)$ & & & 20.8445 & $(62.9050)$ \\
\hline$M$ & & & 0.0622 & & & & 0.0665 & \\
\hline Likelihood & -277.7229 & & -251.7737 & & -279.6187 & & -278.5857 & \\
\hline Default & 0.0579 & & 0.0979 & & 0.0898 & & 0.0912 & \\
\hline
\end{tabular}

\begin{tabular}{|c|c|c|c|c|c|c|c|c|}
\hline Year & \multicolumn{4}{|c|}{2013} & \multicolumn{4}{c|}{2014} \\
\hline Model & \multicolumn{2}{|c|}{ GBM } & \multicolumn{2}{|c|}{ Shot Noise } & \multicolumn{2}{c|}{ GBM } & \multicolumn{2}{c|}{ Shot Noise } \\
\hline$\mu$ & 0.0614 & $(0.0311)$ & 0.0312 & $(0.3772)$ & 0.0683 & $(0.0138)$ & 0.0648 & $(0.0258)$ \\
\hline$\sigma$ & 0.0440 & $(0.0083)$ & 0.0334 & $(0.0076)$ & 0.0342 & $(0.0054)$ & 0.0136 & $(0.0188)$ \\
\hline$\mu_{2} \rho$ & & & 0.0004 & $(0.0006)$ & & & 0.0012 & $(0.0009)$ \\
\hline$Z_{0}$ & & & 9.6337 & $(8.1758)$ & & & 3.0538 & $(1.4439)$ \\
\hline$\delta$ & & & 8.0597 & $(3.0248)$ & & & 10.3560 & $(1.9054)$ \\
\hline$M$ & & & 0.0392 & & & & 0.0377 & \\
\hline Likelihood & -273.6271 & & -269.8824 & & -269.8306 & & -267.1420 & \\
\hline Default & 0.0003 & & 0.0000 & & 0.0000 & & 0.0000 & \\
\hline
\end{tabular}


Table 10: Model Parameters for Citi

\begin{tabular}{|c|c|c|c|c|c|c|c|c|}
\hline Year & \multicolumn{4}{|c|}{2011} & \multicolumn{4}{c|}{2012} \\
\hline Model & \multicolumn{2}{|c|}{ GBM } & \multicolumn{2}{c|}{ Shot Noise } & \multicolumn{2}{c|}{ GBM } & \multicolumn{2}{c|}{ Shot Noise } \\
\hline$\mu$ & 0.0794 & $(0.0546)$ & 0.1663 & $(0.1016)$ & 0.0126 & $(0.0418)$ & -0.0412 & $(0.0480)$ \\
\hline$\sigma$ & 0.1685 & $(0.0226)$ & 0.0982 & $(0.0406)$ & 0.0808 & $(0.0176)$ & 0.0497 & $(0.0117)$ \\
\hline$\mu_{2} \rho$ & & & 0.0081 & $(0.0153)$ & & & 0.0006 & $(0.0015)$ \\
\hline$Z_{0}$ & & & -8.6396 & $(3.5270)$ & & & 17.7945 & $(24.4732)$ \\
\hline$\delta$ & & & 6.9651 & $(1.7011)$ & & & 5.5609 & $(2.7909)$ \\
\hline$M$ & & & 0.1332 & & & & 0.0550 & \\
\hline Likelihood & -275.1723 & & -270.9593 & & -276.3922 & & -271.5470 & \\
\hline Default & 0.2473 & & 0.4233 & & 0.2851 & & 0.0038 & \\
\hline
\end{tabular}

\begin{tabular}{|c|c|c|c|c|c|c|c|c|}
\hline Year & \multicolumn{5}{|c|}{2013} & \multicolumn{4}{c|}{2014} \\
\hline Model & \multicolumn{2}{|c|}{ GBM } & \multicolumn{2}{c|}{ Shot Noise } & \multicolumn{2}{c|}{ GBM } & \multicolumn{2}{c|}{ Shot Noise } \\
\hline$\mu$ & -0.0018 & $(0.3092)$ & -0.0188 & $(0.3041)$ & 0.0452 & $(0.0292)$ & 0.0259 & $(0.0254)$ \\
\hline$\sigma$ & 0.0581 & $(0.0405)$ & 0.0525 & $(0.0509)$ & 0.0385 & $(0.0066)$ & 0.0301 & $(0.0055)$ \\
\hline$\mu_{2} \rho$ & & & 0.0011 & $(0.0028)$ & & & 0.0001 & $(0.0003)$ \\
\hline$Z_{0}$ & & & 1.9977 & $(0.4341)$ & & & 17.1031 & $(21.8423)$ \\
\hline$\delta$ & & & 10.3581 & $(3.4093)$ & & & 9.0585 & $(3.7260)$ \\
\hline$M$ & & & 0.0619 & & & & 0.0323 & \\
\hline Likelihood & -271.8972 & & -271.1865 & & -266.9866 & & -262.7237 & \\
\hline Default & 0.1188 & & 0.1066 & & 0.0001 & & 0.0000 & \\
\hline
\end{tabular}


Table 11: Model Parameters for BAC

\begin{tabular}{|c|c|c|c|c|c|c|c|c|}
\hline Year & \multicolumn{4}{|c|}{2011} & \multicolumn{4}{c|}{2012} \\
\hline Model & \multicolumn{2}{|c|}{ GBM } & \multicolumn{2}{c|}{ Shot Noise } & \multicolumn{2}{c|}{ GBM } & \multicolumn{2}{c|}{ Shot Noise } \\
\hline$\mu$ & 0.0858 & $(0.0957)$ & -0.3241 & $(1.2216)$ & -0.0497 & $(0.0522)$ & -0.1429 & $(1.4609)$ \\
\hline$\sigma$ & 0.1002 & $(0.0270)$ & 0.0757 & $(0.0187)$ & 0.0722 & $(0.0154)$ & 0.0401 & $(0.0081)$ \\
\hline$\mu_{2} \rho$ & & & 0.0004 & $(0.0005)$ & & & 0.0002 & $(0.0006)$ \\
\hline$Z_{0}$ & & & 65.0639 & $(187.5720)$ & & & 32.5817 & $(49.6565)$ \\
\hline$\delta$ & & & 0.5743 & $(1.6504)$ & & & 4.4704 & $(1.7148)$ \\
\hline$M$ & & & 0.0783 & & & & 0.0427 & \\
\hline Likelihood & -279.0902 & & -264.9633 & & -277.3099 & & -269.8235 & \\
\hline Default & 0.0684 & & 0.0004 & & 0.6767 & & 0.1249 & \\
\hline
\end{tabular}

\begin{tabular}{|c|c|c|c|c|c|c|c|c|}
\hline Year & \multicolumn{4}{|c|}{2013} & \multicolumn{4}{c|}{2014} \\
\hline Model & \multicolumn{2}{|c|}{ GBM } & \multicolumn{2}{|c|}{ Shot Noise } & \multicolumn{2}{c|}{ GBM } & \multicolumn{2}{c|}{ Shot Noise } \\
\hline$\mu$ & 0.0113 & $(0.0409)$ & 0.0014 & $(0.0388)$ & 0.0557 & $(0.0252)$ & 0.0090 & 0.2299 \\
\hline$\sigma$ & 0.0613 & $(0.0122)$ & 0.0480 & $(0.0133)$ & 0.0357 & $(0.0042)$ & 0.0217 & 0.0134 \\
\hline$\mu_{2} \rho$ & & & 0.0016 & $(0.0022)$ & & & 0.0002 & 0.0005 \\
\hline$Z_{0}$ & & & 0.2613 & $(0.2622)$ & & & 17.3249 & 20.2392 \\
\hline$\delta$ & & & 11.0773 & $(0.6166)$ & & & 4.0714 & 3.1536 \\
\hline$M$ & & & 0.0622 & & & & 0.0267 & \\
\hline Likelihood & -272.8862 & & -270.9900 & & -267.2663 & & -259.9658 & \\
\hline Default & 0.0987 & & 0.0745 & & 0.0000 & & 0.0000 & \\
\hline
\end{tabular}




\section{Conclusion}

In this paper our main focus is on the simultaneous default probabilities during 2004-2010, since this includes subprime mortgage crisis. If we look at the retrieved equity graphs, the shot noise model performed better than simple GBM model for 2008 (Citi), 2009, and 2010. For 2008, we obtained higher default probability for Citi and also, concluded that during 2006-2007 there were some warnings about upcoming crisis for this company. For 2009, we obtained better fit with shot noise model for JPM and BAC. For Citi, reported GBM and the shot noise fits were similar. In general, simultaneous default probabilities were higher for shot noise model than for GBM. For 2010, shot noise model gave better fit and higher default probabilities for all company combinations. In 2013, the probability of all three companies defaulting at the same time goes to 0 . Simultaneous default probabilities are 0 for all company combinations in 2014 and 2015.

Looking at the retrieved equity graphs for 2007-2008 (Graphs 5 and 13) and 2008-2009 (Graphs 6, 10, and 14), we can say that shot noise model outperforms GBM during bad economy. During the period of upward trends in equity, we cannot say that shot noise model outperforms GBM model; however, in some cases we get better fit from the shot noise model (see Graphs 4, 20, 23, and 26).

Our model did predict higher simultaneous default probabilities for the years of 2009 and 2010, and this shows that GBM model underestimates default probability predictions for these years, compared to our model. However, we still do not know to what extent the default probability estimates from our model are close to reality. Therefore, we use market data to check form a different perspective which model is better, GBM model or our model.

We used Senior 5 year CDS Spread Mid data from Thomson Reuters (datasource-CMA) for JPM, Citi, and BAC. We call this data CDS spread. The data is provided in USD. Since we have been using monthly data in our previous calculations, we will also use monthly CDS spreads. The data is available only until 2010/09/30; therefore, we have 70 monthly observations for each company starting at 2004/12/30. Figure 1 illustrates the dynamics of CDS spreads. 


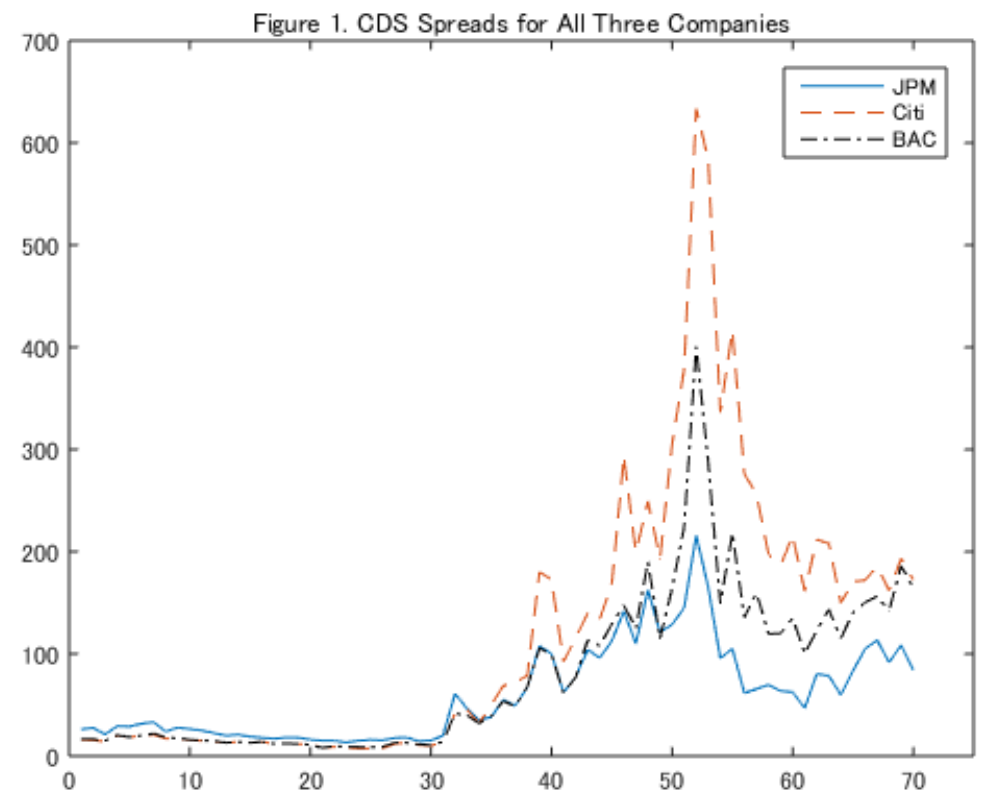

Observations 25-49 are from 2006/12/30-2008/12/30. We can see that CDS spreads for Citi and BAC increased greatly in 2009. To check the connection between CDS spreads and asset values, we first simulated the asset processes for both models. We will illustrate this by an example. Using 2005-2006 data, we already estimated parameters and used them to predict default at the end of 2007. Rather than predicting, we now used 2005-2006 parameters to simulate asset processes for 2005-2006. We retrieved initial asset value from Black-Scholes equation using just variance parameter and then simulated asset paths 50,000 times and took average. Using 2006-2007 data, we simulated asset paths for 2006-2007 in a similar manner. We took average of asset values for overlapping periods (e.g. for the year of 2006 in this example). By putting together these paths, we obtained times series of asset values from both models. Then, using R 3.1.3 we regressed 2004/12/30-2010/09/30 CDS spreads of each company on all three asset time series of 2004/12/30-2010/09/30. JMPcds, Citicds, and BACcds are CDS spread data vectors. JPMgbm, Citigbm, and BACgbm are asset vectors simulated from GBM model. JPMshotnoise, Citishotnoise, and BACshotnoise are asset vectors simulated from our model. For each company's CDS spread, we did two regressions: one based on assets simulated from GBM model and another based on assets simulated from our model. Regression equations are as follows:

$$
\begin{gathered}
J P M c d s=\alpha_{1} J P M g b m+\beta_{1} \text { Citigbm }+\gamma_{1} B A C g b m+\epsilon_{J P M} \\
J P M c d s=\alpha_{1}^{\star} J P M \text { shotnoise }+\beta_{1}^{\star} \text { Citishotnoise }+\gamma_{1}^{\star} \text { BACshotnoise }+\epsilon_{J P M}^{\star}
\end{gathered}
$$




$$
\begin{gathered}
\text { Citicds }=\alpha_{2} J P M g b m+\beta_{2} \text { Citigbm }+\gamma_{2} \text { BACgbm }+\epsilon_{C i t i} \\
\text { Citicds }=\alpha_{2}^{\star} J P M \text { shotnoise }+\beta_{2}^{\star} \text { Citishotnoise }+\gamma_{2}^{\star} \text { BACshotnoise }+\epsilon_{\text {Citi }}^{\star} \\
\text { BACcds }=\alpha_{3} J P M g b m+\beta_{3} \text { Citigbm }+\gamma_{3} B A C g b m+\epsilon_{B A C} \\
\text { BACcds }=\alpha_{3}^{\star} J P M \text { shotnoise }+\beta_{3}^{\star} \text { Citishotnoise }+\gamma_{3}^{\star} \text { BACshotnoise }+\epsilon_{B A C}^{\star}
\end{gathered}
$$

From the tables below, we can see that asset values simulated from our model are able to explain CDS

\begin{tabular}{|c|c|c|}
\hline & \multicolumn{2}{|c|}{ Dependent variable: } \\
\hline & \multicolumn{2}{|c|}{ JPMcds } \\
\hline & (1) & $(2)$ \\
\hline JPMgbm & $\begin{array}{c}0.0003^{* * *} \\
(0.0001)\end{array}$ & \\
\hline Citigbm & $\begin{array}{c}0.0001 \\
(0.0001)\end{array}$ & \\
\hline BACgbm & $\begin{array}{c}-0.0003^{*} \\
(0.0002)\end{array}$ & \\
\hline JPMshotnoise & & $\begin{array}{l}0.001^{* * *} \\
(0.0001)\end{array}$ \\
\hline Citishotnoise & & $\begin{array}{l}0.0001^{* * *} \\
(0.00004)\end{array}$ \\
\hline BACshotnoise & & $\begin{array}{c}-0.001^{\text {*** }} \\
(0.0001)\end{array}$ \\
\hline Observations & 70 & 70 \\
\hline $\mathrm{R}^{2}$ & 0.850 & 0.854 \\
\hline Adjusted $\mathrm{R}^{2}$ & 0.843 & 0.847 \\
\hline Residual Std. Error $(\mathrm{df}=67)$ & 29.915 & 29.517 \\
\hline F Statistic $(\mathrm{df}=3 ; 67)$ & $126.537^{* * *}$ & $130.582^{* * *}$ \\
\hline
\end{tabular}
spreads better than asset values simulated from GBM model. If we use asset values from our model, more regression parameters will be statistically significant.

Table 12: Regression Results for JPM CDS Spread 
Table 13: Regression Results for Citi CDS Spread

\begin{tabular}{lcc}
\hline \hline & \multicolumn{2}{c}{ Dependent variable: } \\
\cline { 2 - 3 } & $(1)$ & $(2)$ \\
\hline JPMgbm & $0.001^{* *}$ & \\
& $(0.0003)$ & \\
Citigbm & -0.0001 & \\
& $(0.0002)$ & \\
BACgbm & -0.0004 & \\
& $(0.0005)$ & \\
JPMshotnoise & & $0.001^{* * *}$ \\
& & $(0.0003)$ \\
Citishotnoise & & -0.0001 \\
& & $(0.0001)$ \\
BACshotnoise & & $-0.001^{*}$ \\
& & $(0.0004)$ \\
\hline Observations & & 70 \\
$\mathrm{R}^{2}$ & & 0.797 \\
Adjusted $\mathrm{R}^{2}$ & & 0.788 \\
Residual Std. Error $(\mathrm{df}=67)$ & 83.428 & 82.573 \\
F Statistic $(\mathrm{df}=3 ; 67)$ & $85.634^{* * *}$ & $87.883^{* * *}$ \\
\hline \hline Note: & ${ }^{*} \mathrm{p}<0.1 ;{ }^{* *} \mathrm{p}<0.05 ;{ }^{* * *} \mathrm{p}<0.01$ \\
& &
\end{tabular}


Table 14: Regression Results for BAC CDS Spread

\begin{tabular}{lcc}
\hline \hline & \multicolumn{2}{c}{ Dependent variable: } \\
\cline { 2 - 3 } & \multicolumn{2}{c}{ BACcds } \\
\hline JPMgbm & $(1)$ & $(2)$ \\
& $\left(0.0004^{* * *}\right.$ & \\
Citigbm & -0.00005 & \\
& $(0.0001)$ & \\
BACgbm & -0.0003 & \\
& $(0.0002)$ & \\
JPMshotnoise & & $0.001^{* * *}$ \\
& & $(0.0002)$ \\
Citishotnoise & & -0.00003 \\
& & $(0.0001)$ \\
BACshotnoise & & $-0.0005^{* * *}$ \\
& & $(0.0002)$ \\
\hline Observations & & 70 \\
$\mathrm{R}^{2}$ & & 0.860 \\
Adjusted $\mathrm{R}^{2}$ & & 0.854 \\
Residual Std. Error $(\mathrm{df}=67)$ & 42.242 & 42.644 \\
F Statistic $(\mathrm{df}=3 ; 67)$ & $140.054^{* * *}$ & $137.009^{* * *}$ \\
\hline \hline Note: & ${ }^{*} \mathrm{p}<0.1 ;{ }^{* *} \mathrm{p}<0.05 ;{ }^{* * *} \mathrm{p}<0.01$ \\
& &
\end{tabular}


We also display fitted CDS spread values (fitted values from above regressions) together with real CDS spread data below. We can see that fitted values based on our model's asset time series provide better approximation of the real data than the values based on GBM model. It is also obvious that simple regression model cannot explain the spike in CDS spreads in 2009. For this, we might need to consider self-exciting processes, such as Hawkes process.
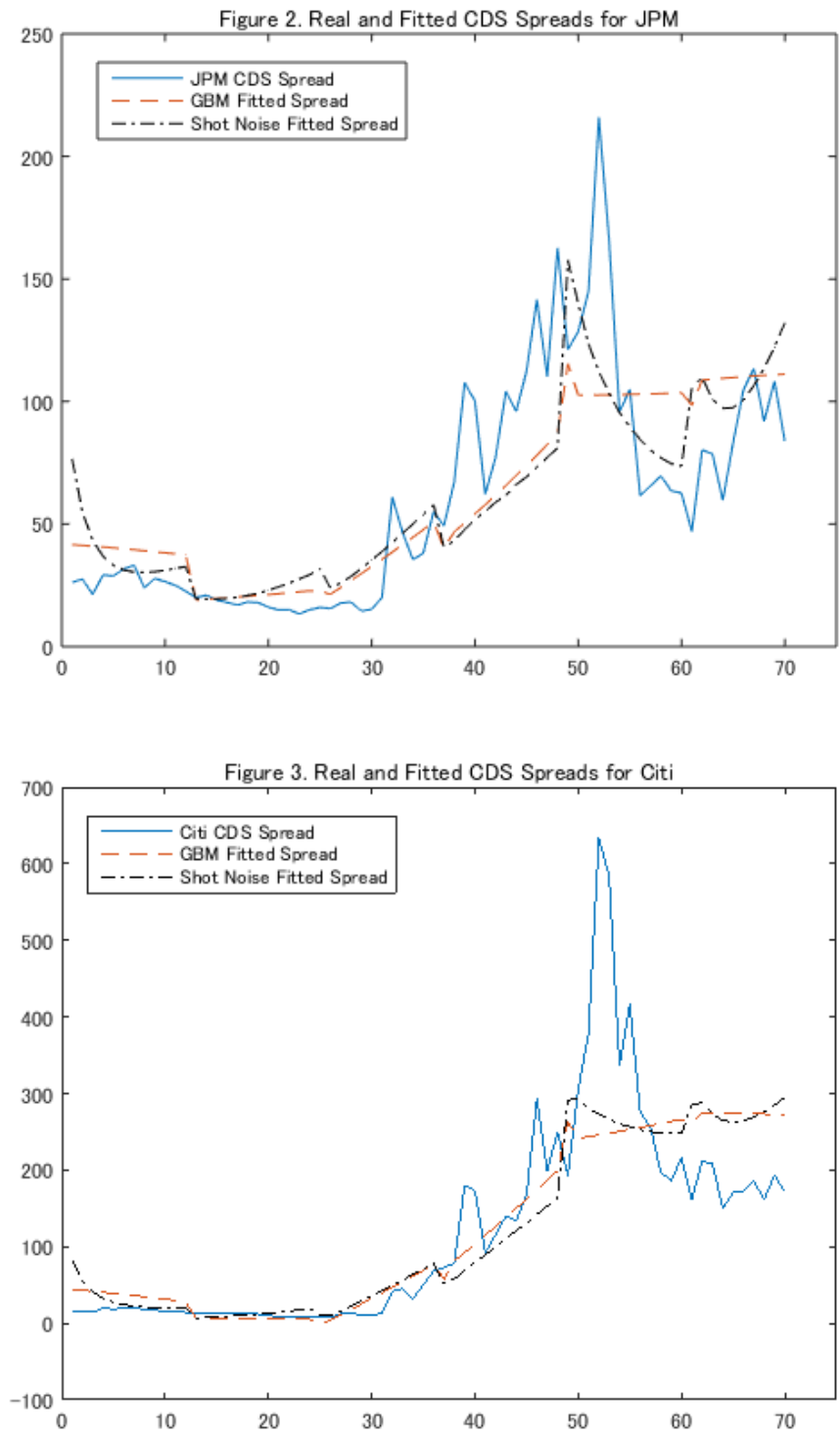


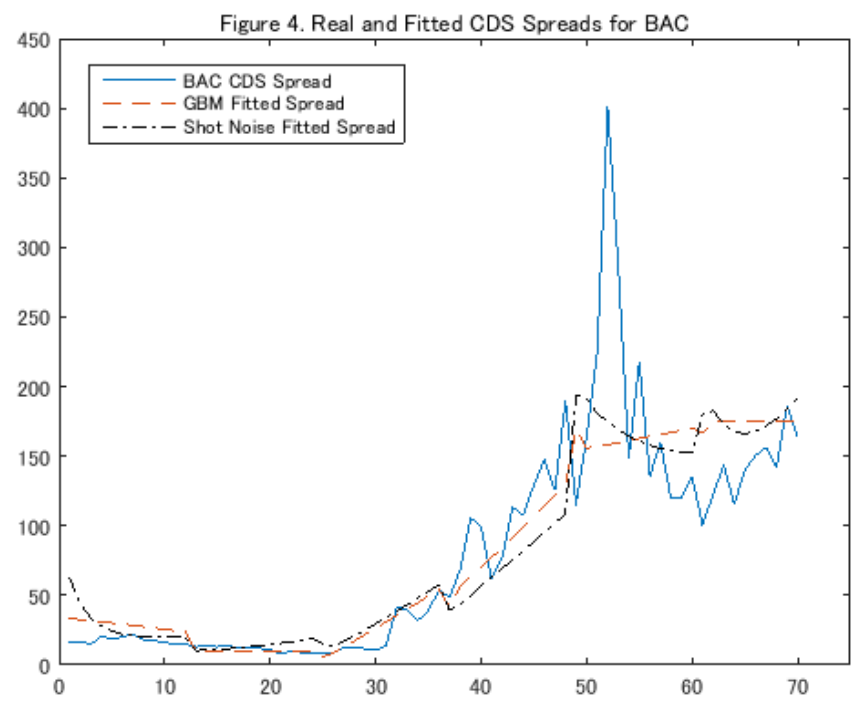

Finally, we display DCC Garch Model's estimates of dynamic conditional correlation between logarithmic CDS spread returns. We again used $\mathrm{R}$ for the analysis. We first estimated VAR model (setting type= "const") for asset return vectors and then applied dcc.estimation function (setting model="diagonal") to the residuals. We selected VAR lag order by VARselect function where we set lag.max to default value 10. We applied Garch $(1,1)$ model to each residual vector and used those parameters as initial values for dcc.estimation.

In case of Citi, we obtained one negative fitted CDS spread from GBM model assets; therefore, we cannot take logarithm. Negative spread means that the fit is not good. We exclude Citi from the analysis and focus on JPM and BAC. Lag order 1 was selected in all (real, GBM, and shot noise) cases. Figure 5 displays dynamic conditional correlations for real CDS spread returns and for the returns based on fitted CDS spreads from GBM and our model (these are fitted values from the regression on assets that we explained above). We again see that our model provides a better approximation to the dynamic conditional correlation of the real data. 


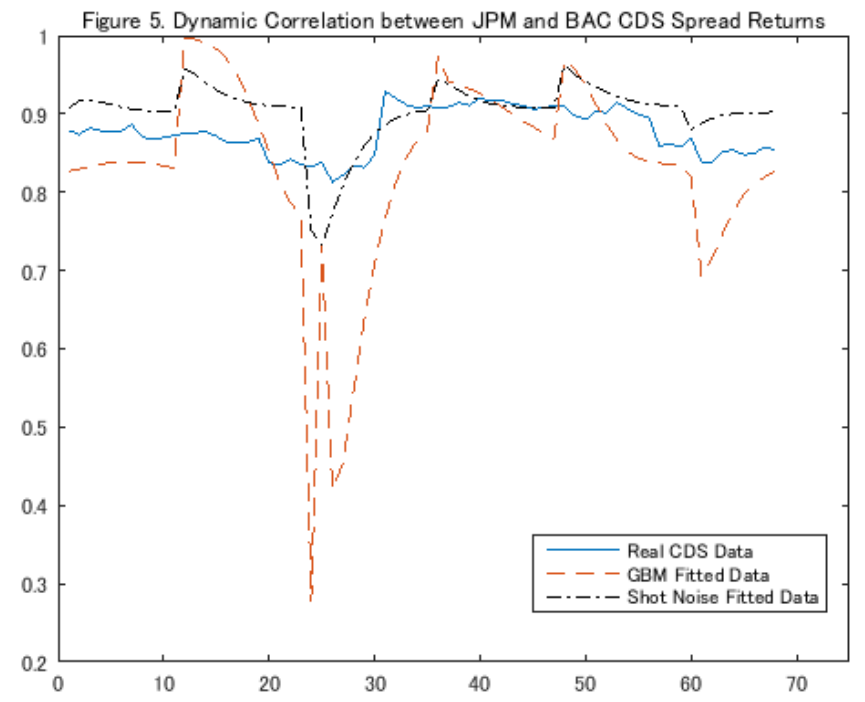

We can conclude that asset values simulated from our model explain characteristics of real CDS spread data better than asset values simulated from GBM model. Therefore, we can say that our default probability predictions are also closer to reality. For future research, we would like to expand this model and use it in the analysis of more than three companies. We anticipate that adding additional companies to the model will not increase computational burden since we will be using only one shot noise process. Also, we would like to analyze simultaneous defaults in different industries. For this, we consider using more than one shot noise model to incorporate industry dependencies in the model. 


\section{References}

[1] Black, F. and M. Scholes (1973) "The pricing of options and corporate liabilities," The Journal of Political Economy 81(3), pp.637-654.

[2] Dalla Valle, L., M.E. De Giuli, C. Tarantola, and C. Manelli (2016) "Default probability estimation via pair copula constructions," European Journal of Operational Research 249(1), pp.298-311.

[3] Dassios, A. and J.W. Jang (2005) "Kalman-Bucy filtering for linear systems driven by the Cox process with shot noise intensity and its application to the pricing of reinsurance contracts," Journal of Applied Probability 42(1), pp.93-107.

[4] Dong, Y., G. Wang, and K. C. Yuen (2014) "Bilateral counterparty risk valuation on a CDS with a common shock model," Methodology and Computing in Applied Probability 16(3), pp.643-673.

[5] Duan, J.C. (1994) "Maximum likelihood estimation using price data of the derivative contract," Mathematical Finance 4(2), pp.155-167.

[6] Duan, J.C. (2000) "Correction: Maximum likelihood estimation using price data of the derivative contract," Mathematical Finance 10(4), pp.461-462.

[7] Duan, J.C., G. Gauthier, and J.G. Simonato (2004) "On the equivalence of the KMV and maximum likelihood methods for structural credit risk models".

(http://www.rmi.nus.edu.sg/duanjc/index_files/files/KMV-MLE_Nov\%2030\%202004.pdf accessed on $2015 / 12 / 19)$

[8] Filiz, I.O., X. Guo, J. Morton, and B. Sturmfels (2012) "Graphical models for correlated defaults," Mathematical Finance 22(4), pp.621-644.

[9] Freed, D. (2011, December 14) Bank of America 2012: The Worst is Yet to Come? TheStreet.CNBC. (http://www.cnbc.com/id/45673978 accessed on 2015/12/19)

[10] Giesecke, K. (2004) "Correlated default with incomplete information," Journal of Banking and Finance 28(7), pp.1521-1545.

[11] Giesecke, K., L.R. Goldberg, and X. Ding (2011) "A top-down approach to multiname credit," Operations Research 59(2), pp.283-300.

[12] Herbertsson, A., J. Jang, and T. Schmidt (2011) "Pricing basket default swaps in a tractable shot noise model," Statistics and Probability Letters 81(8), pp.1196-1207.

[13] Jang, J. and A. Dassios (2013) "A bivariate shot noise self-exciting process for insurance," Insurance: Mathematics and Economics 53(3), pp.524-532. 
[14] Karatzas, I. and S.E. Shreve (1998) Brownian Motion and Stochastic Calculus, Springer Science+Business Media, 2nd edition.

[15] Kunisch, M. and M. Uhrig-Homburg (2008) " Modeling simultaneous defaults: A top-down approach," Journal of Fixed Income 18(1), pp.25-36.

[16] Landau, H.J. and L. A. Shepp (1970) "On the supremum of a Gaussian process," The Indian Journal of Statistics, Series A 32(4), pp.369-378.

[17] Lehar, A. (2005) "Measuring systemic risk: A risk management approach," Journal of Banking and Finance 29(10), pp.2577-2603.

[18] Liang, X. and G. Wang (2012) "On a reduced form credit risk model with common shock and regime switching," Insurance: Mathematics and Economics 51(3), pp.567-575.

[19] Ma, Y.K. and J.H. Kim (2010) "Pricing the credit default swap rate for jump diffusion default intensity processes," Quantitative Finance 10(8), pp.809-817.

[20] Merton, R.C. (1974) "On the pricing of corporate debt: the risk structure of interest rates," The Journal of Finance 29(2), pp.449-470.

[21] Pianeti, R., R. Giacometti, and V. Acerbis (2012) "Estimating the joint probability of default using credit default swap and bond data," Journal of Fixed Income 21(3), pp.44-58.

[22] Rucker, P. and J. Stempel (2009, January 16) Bank of America gets big government bailout. Reuters. (http://www.reuters.com/article/us-banks-idUSTRE50F1Q720090116 accessed on 2015/12/19)

[23] Salmon, F. (2009, February 23) Recipe for Disaster: The Formula That Killed Wall Street. Wired. (http://www.wired.com/2009/02/wp-quant/ accessed on 2015/12/19)

[24] Schmidt, T. (2014) "Catastrophe insurance modeled by shot-noise processes," Risks 2(1), pp.3-24.

[25] Shreve, S.E. (2004) Stochastic Calculus for Finance II Continuous-Time Models, Springer-Verlag.

[26] Steele, J.M. (2001) Stochastic Calculus and Financial Applications, Springer-Verlag.

[27] Wilchins, D. and J. Stempel (2008, November 25) Citigroup gets massive government bailout. Reuters. (http://www.reuters.com/article/us-citigroup-idUSTRE4AJ45G20081125 accessed on 2015/12/19) 


\section{Appendices}

\section{A Mathematical Proofs}

\section{Dynamics of $V=\left(V_{t}\right)_{t>0}$ under approximation (Section 1.2)}

First, we will prove that $Z_{t}=Z_{0} e^{-\delta t}+\sqrt{2 \delta} \int_{0}^{t} e^{-\delta(t-s)} d B_{s}^{(2)}$ is a semimartingale. Applying Itô's formula to $e^{\delta t} B_{t}^{(2)}$, we get

$$
e^{\delta t} B_{t}^{(2)}=\int_{0}^{t} \delta e^{\delta s} B_{s}^{(2)} d s+\int_{0}^{t} e^{\delta s} d B_{s}^{(2)}
$$

Multiplying both sides by $e^{-\delta t}$, we have

$$
\begin{gathered}
B_{t}^{(2)}=\int_{0}^{t} \delta e^{-\delta(t-s)} B_{s}^{(2)} d s+\int_{0}^{t} e^{-\delta(t-s)} d B_{s}^{(2)} \\
\longrightarrow \int_{0}^{t} e^{-\delta(t-s)} d B_{s}^{(2)}=B_{t}^{(2)}-\int_{0}^{t} \delta e^{-\delta(t-s)} B_{s}^{(2)} d s
\end{gathered}
$$

After substituting this into the equation of $Z_{t}$, we get

$$
\begin{aligned}
Z_{t} & =Z_{0} e^{-\delta t}+\sqrt{2 \delta} B_{t}^{(2)}-\sqrt{2 \delta} \int_{0}^{t} \delta e^{-\delta(t-s)} B_{s}^{(2)} d s \\
& =Z_{0}+\left(Z_{0} e^{-\delta t}-Z_{0}-\sqrt{2 \delta} \int_{0}^{t} \delta e^{-\delta(t-s)} B_{s}^{(2)} d s\right)+\sqrt{2 \delta} B_{t}^{(2)}
\end{aligned}
$$

The process inside the brackets starts at 0 . For any fixed $t$, the integral inside the brackets is a welldefined Lebesgue-Stietljes integral and as a function of $t$, it is of bounded variation (Karatzas \& Shreve, Remark 4.6(i) $\left[14\right.$, p. 23, 150]). This means that $Z_{t}$ can be expressed as a sum of the initial value, the bounded variation process and the local martingale. Hence, it is a semimartingale. Also, we have

$$
\langle Z\rangle_{t}=2 \delta t
$$

Next, we show the dynamics of the asset value.

$$
\begin{gathered}
V_{t}=e^{X_{0}+\left(\mu-\frac{1}{2} \sigma^{2}\right) t+\sigma B_{t}^{(1)}-\frac{\mu_{1} \rho}{\delta}-Z_{t} \sqrt{\frac{\mu_{2} \rho}{2 \delta}}} \\
d V_{t}=V_{t}\left(\mu-\frac{1}{2} \sigma^{2}\right) d t+V_{t} \sigma d B_{t}^{(1)}+\frac{1}{2} V_{t} \sigma^{2} d t-V_{t} \sqrt{\frac{\mu_{2} \rho}{2 \delta}} d Z_{t}+\frac{1}{2} V_{t} \frac{\mu_{2} \rho}{2 \delta} d\langle Z\rangle_{t} \\
=V_{t} \mu d t+V_{t} \sigma d B_{t}^{(1)}-V_{t} \sqrt{\frac{\mu_{2} \rho}{2 \delta}}\left(-\delta Z_{t} d t+\sqrt{2 \delta} d B_{t}^{(2)}\right)+\frac{1}{2} V_{t} \frac{\mu_{2} \rho}{2 \delta} 2 \delta d t \\
=V_{t}\left(\mu+\frac{1}{2} \mu_{2} \rho+\delta \sqrt{\frac{\mu_{2} \rho}{2 \delta}} Z_{t}\right) d t+V_{t} \sigma d B_{t}^{(1)}-V_{t} \sqrt{\mu_{2} \rho} d B_{t}^{(2)}
\end{gathered}
$$


2. $V=\left(V_{t}\right)_{t \geq 0}$ is a semimartingale (Section 1.2)

$$
V_{t}=V_{0}+\int_{0}^{t} V_{s} Q_{s} d s+\int_{0}^{t} V_{s} \sigma d B_{s}^{(1)}-\int_{0}^{t} V_{s} \sqrt{\mu_{2} \rho} d B_{s}^{(2)}
$$

We are looking at time up to fixed $T$. The first integral is again a Lebesgue-Stieltjes integral and is a bounded variation process. The second and third integrals are local martingales since $P\left[\int_{0}^{T} V_{s}^{2} \sigma^{2} d s<\infty\right]=$ 1 and $P\left[\int_{0}^{T} V_{s}^{2} \mu_{2} \rho d s<\infty\right]=1$. Their sum is again a local martingale. Therefore, $V_{t}$ is a semimartingale for $t \in[0, T]$.

3. The condition for measure change is satisfied (Section 1.3.1)

$$
\begin{gathered}
H_{t}(\theta)=e^{\sum_{i=1}^{2} \int_{0}^{t} \theta_{s}^{i} d B_{s}^{i}-\frac{1}{2} \int_{0}^{t}\left\|\theta_{s}\right\|^{2} d s} \\
\theta_{t}^{1}=\frac{r-Q_{t}+\sqrt{\mu_{2} \rho}}{\sigma} \\
\theta_{t}^{2}=1
\end{gathered}
$$

We have to show that Novikov's condition $E\left[e^{\frac{1}{2} \int_{0}^{T}\left\|\theta_{t}\right\|^{2} d t}\right]<\infty$ is satisfied. Let $K=r-\mu-\frac{\mu_{2} \rho}{2}+\sqrt{\mu_{2} \rho}$.

$$
\begin{gathered}
\frac{1}{2}\left\|\theta_{t}\right\|^{2}=\frac{\left(r-\mu-\frac{\mu_{2} \rho}{2}+\sqrt{\mu_{2} \rho}-\delta \sqrt{\frac{\mu_{2} \rho}{2 \delta}} Z_{t}\right)^{2}}{2 \sigma^{2}}+\frac{1}{2}=\frac{\left(K-\delta \sqrt{\frac{\mu_{2} \rho}{2 \delta}} Z_{t}\right)^{2}}{2 \sigma^{2}}+\frac{1}{2} \\
\frac{1}{2}\left\|\theta_{t}\right\|^{2} \leq \frac{1}{2}+\frac{\left(|K|+\left|\delta \sqrt{\frac{\mu_{2} \rho}{2 \delta}} Z_{0} e^{-\delta t}\right|+\delta \sqrt{\mu_{2} \rho}\left|\int_{0}^{t} e^{-\delta(t-s)} d B_{s}^{(2)}\right|\right)^{2}}{2 \sigma^{2}}
\end{gathered}
$$

Now, $e^{-\delta t} \leq 1$ and $|K|+\left|\delta \sqrt{\frac{\mu_{2} \rho}{2 \delta}} Z_{0} e^{-\delta t}\right| \leq|K|+\left|\delta \sqrt{\frac{\mu_{2} \rho}{2 \delta}} Z_{0}\right|=C$ where $\mathrm{C}$ is a positive constant. Also,

$$
\left|\int_{0}^{t} e^{-\delta(t-s)} d B_{s}^{(2)}\right| \leq\left|\int_{0}^{t} 1 d B_{s}^{(2)}\right|=\left|B_{t}^{(2)}\right|
$$

So,

$$
\frac{1}{2}\left\|\theta_{t}\right\|^{2} \leq \frac{1}{2}+\frac{\left(C+\delta \sqrt{\mu_{2} \rho}\left|B_{t}^{(2)}\right|\right)^{2}}{2 \sigma^{2}}
$$

Let $B_{T}^{*}=\sup _{0 \leq t \leq T}\left|B_{t}^{(2)}\right|$. Using Corollary 5.14 from Karatzas and Shreve [14, p. 199], we take any sequence that satisfies $0=t_{0}<t_{1}<\ldots<t_{n} \rightarrow \infty$. Now,

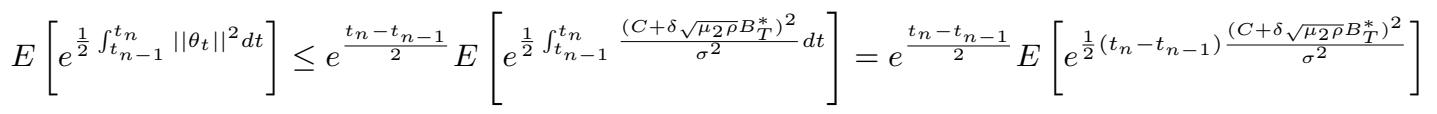

$\frac{C+\delta \sqrt{\mu_{2} \rho} B_{t}^{(2)}}{\sqrt{2} \sigma}$ is a Gaussian process. For each $\omega$, Brownian motion is continuous in $t$; therefore, its maximum on $[0, T]$ exists. From Burkholder-Davis-Gundy inequality (Karatzas \& Shreve [14, p. 166]), 
there exists some constant $K_{\frac{1}{2}}$ and $E\left[\sup _{0 \leq t \leq T}\left|B_{t}\right|\right] \leq K_{\frac{1}{2}} \sqrt{T}$.

$$
\rightarrow \sup _{0 \leq t \leq T}\left|\frac{C+\delta \sqrt{\mu_{2} \rho} B_{t}^{(2)}}{\sqrt{2} \sigma}\right|<\infty \quad \text { a.s. }
$$

Then, by Landau and Shepp [16], there exists some $\varepsilon>0$, such that

$$
E\left[e^{\varepsilon \frac{\left(C+\delta \sqrt{\mu_{2} \rho} B_{T}^{*}\right)^{2}}{2 \sigma^{2}}}\right]<\infty
$$

We make the interval $t_{n}-t_{n-1} \forall n \geq 1$ equal to $\varepsilon$. Then,

$$
E\left[e^{\left(t_{n}-t_{n-1}\right) \frac{\left(C+\delta \sqrt{\mu_{2} \rho} B_{T}^{*}\right)^{2}}{2 \sigma^{2}}}\right]<\infty \quad \forall n \geq 1
$$

This means that

$$
E\left[e^{\frac{1}{2} \int_{t_{n}-1}^{t_{n}} \theta_{t}^{2} d t}\right]<\infty \quad \forall n \geq 1
$$

and from the above mentioned Corollary 5.14, we can say that $H_{t}(\theta)$ is a martingale.

The idea of this proof was borrowed from Nate Eldredge.

http://math.stackexchange.com/questions/133691/can-i-apply-the-girsanov-theorem-to-an-ornstein-

uhlenbeck-process accessed on 10/18/15

Because $H_{t}(\theta)$ is a martingale, we can change the measure and $\tilde{B}_{t}$ becomes 2-dimensional Brownian motion under $\tilde{P}_{T}$.

$\tilde{W}_{t}=\frac{\sigma}{M} \tilde{B}_{t}^{(1)}-\frac{\sqrt{\mu_{2} \rho}}{M} \tilde{B}_{t}^{(2)}$ is a martingale and its quadratic variation is $t$. Therefore, it is a standard Brownian motion under $\tilde{P}_{T}$.

$d\left(e^{-r t} V_{t}\right)=e^{-r t} V_{t} M d \tilde{W}_{t}$ and $e^{-r t} V_{t}$ is a local martingale under $\tilde{P}_{T}$. However, we also have

$$
\tilde{E}\left[e^{\frac{1}{2} \int_{0}^{T} M^{2} d t}\right]<\infty
$$

and from proposition 14.2 in Steele [26, p.241] we conclude that $e^{-r t} V_{t}$ is a martingale under $\tilde{P}_{T}$.

4. The condition for measure change in the multidimensional model is satisfied (Section 1.3.2) Using the above results,

$$
\frac{1}{2}\left\|\theta_{t}\right\|^{2} \leq \sum_{j=1}^{m+1}\left[\frac{1}{2}+\frac{\left(C^{j}+\delta^{j} \sqrt{\mu_{2}^{j} \rho^{j}}\left|B_{t}^{(m+1)}\right|\right)^{2}}{2\left(\sigma^{j}\right)^{2}}\right.
$$


Let $C=\max _{j} C^{j}, \delta=\max _{j} \delta^{j}, \mu_{2} \rho=\max _{j} \mu_{2}^{j} \rho^{j}, \sigma=\min _{j} \sigma^{j}$. Then,

$$
\frac{1}{2}\left\|\theta_{t}\right\|^{2} \leq(m+1)\left[\frac{1}{2}+\frac{\left(C+\delta \sqrt{\mu_{2} \rho}\left|B_{t}^{(m+1)}\right|\right)^{2}}{2 \sigma^{2}}\right]
$$

Again, we have a Gaussian process and we can use the exactly same reasoning as in Appendix A3.

5. Derivation of the log-likelihood function (Section 1.4) The construction of the log-likelihood function that will be used during estimation is based on the arguments in Duan [5], Duan [6], and Duan, Gauthier, and Simonato [7]. First, we calculate E[ $\left[\ln V_{k \Delta_{t}} \mid V_{(k-1) \Delta_{t}}\right]$ and $\operatorname{Var}\left[\ln V_{k \Delta_{t}} \mid V_{(k-1) \Delta_{t}}\right]$.

$$
\ln \frac{V_{k \Delta_{t}}}{V_{(k-1) \Delta_{t}}}=\left(\mu-\frac{1}{2} \sigma^{2}\right) \Delta_{t}+\sigma\left(B_{k \Delta_{t}}^{(1)}-B_{(k-1) \Delta_{t}}^{(1)}\right)-\sqrt{\frac{\mu_{2} \rho}{2 \delta}}\left(Z_{k \Delta_{t}}-Z_{(k-1) \Delta_{t}}\right)
$$

$Z_{k \Delta_{t}}-Z_{(k-1) \Delta_{t}}$

$$
\begin{aligned}
& =Z_{0} e^{-\delta k \Delta_{t}}+\sqrt{2 \delta} e^{-\delta k \Delta_{t}} \int_{0}^{k \Delta_{t}} e^{\delta s} d B_{s}^{(2)}-Z_{0} e^{-\delta(k-1) \Delta_{t}}-\sqrt{2 \delta} e^{-\delta(k-1) \Delta_{t}} \int_{0}^{(k-1) \Delta_{t}} e^{\delta s} d B_{s}^{(2)} \\
& =Z_{0} e^{-\delta k \Delta_{t}}\left(1-e^{\delta \Delta_{t}}\right)+\sqrt{2 \delta}\left[e^{-\delta k \Delta_{t}} \int_{0}^{k \Delta_{t}} e^{\delta s} d B_{s}^{(2)}-e^{-\delta(k-1) \Delta_{t}} \int_{0}^{(k-1) \Delta_{t}} e^{\delta s} d B_{s}^{(2)}\right] \\
& =Z_{0} e^{-\delta k \Delta_{t}}\left(1-e^{\delta \Delta_{t}}\right)+\sqrt{2 \delta} \\
& {\left[e^{-\delta k \Delta_{t}} \int_{0}^{(k-1) \Delta_{t}} e^{\delta s} d B_{s}^{(2)}+e^{-\delta k \Delta_{t}} \int_{(k-1) \Delta_{t}}^{k \Delta_{t}} e^{\delta s} d B_{s}^{(2)}-e^{-\delta(k-1) \Delta_{t}} \int_{0}^{(k-1) \Delta_{t}} e^{\delta s} d B_{s}^{(2)}\right]} \\
& =Z_{0} e^{-\delta k \Delta_{t}}\left(1-e^{\delta \Delta_{t}}\right)+\sqrt{2 \delta} \\
& {\left[e^{-\delta k \Delta_{t}} \int_{(k-1) \Delta_{t}}^{k \Delta_{t}} d e^{\delta s} d B_{s}^{(2)}+\frac{e^{-\delta(k-1) \Delta_{t}}}{e^{\delta \Delta_{t}}} \int_{0}^{(k-1) \Delta_{t}} e^{\delta s} d B_{s}^{(2)}-e^{-\delta(k-1) \Delta_{t}} \int_{0}^{(k-1) \Delta_{t}} e^{\delta s} d B_{s}^{(2)}\right]} \\
& =Z_{0} e^{-\delta k \Delta_{t}}\left(1-e^{\delta \Delta_{t}}\right)+\sqrt{2 \delta}\left[e^{-\delta k \Delta_{t}} \int_{(k-1) \Delta_{t}}^{k \Delta_{t}} e^{\delta s} d B_{s}^{(2)}+\left(\frac{1}{e^{\delta \Delta_{t}}}-1\right) e^{-\delta(k-1) \Delta_{t}} \int_{0}^{(k-1) \Delta_{t}} e^{\delta s} d B_{s}^{(2)}\right]
\end{aligned}
$$

$$
\begin{aligned}
\ln V_{k \Delta_{t}}-\ln V_{(k-1) \Delta_{t}} & =\left(\mu-\frac{1}{2} \sigma^{2}\right) \Delta_{t}+\sigma\left(B_{k \Delta_{t}}^{(1)}-B_{(k-1) \Delta_{t}}^{(1)}\right)-\sqrt{\frac{\mu_{2} \rho}{2 \delta}} Z_{0} e^{-\delta k \Delta_{t}}\left(1-e^{\delta \Delta_{t}}\right) \\
& -\sqrt{\mu_{2} \rho} e^{-\delta k \Delta_{t}} \int_{(k-1) \Delta_{t}}^{k \Delta_{t}} e^{\delta s} d B_{s}^{(2)}-\sqrt{\mu_{2} \rho}\left(\frac{1}{e^{\delta \Delta_{t}}}-1\right) e^{-\delta(k-1) \Delta_{t}} \int_{0}^{(k-1) \Delta_{t}} e^{\delta s} d B_{s}^{(2)}
\end{aligned}
$$

Given $V_{(k-1) \Delta_{t}}, \ln V_{k \Delta_{t}}$ follows normal distribution.

$$
\begin{gathered}
\mathrm{E}\left[\ln V_{k \Delta_{t}} \mid V_{(k-1) \Delta_{t}}\right]= \\
\ln V_{(k-1) \Delta_{t}}+\left(\mu-\frac{1}{2} \sigma^{2}\right) \Delta_{t}-\sqrt{\frac{\mu_{2} \rho}{2 \delta}} Z_{0} e^{-\delta k \Delta_{t}}\left(1-e^{\delta \Delta_{t}}\right)-\sqrt{\mu_{2} \rho}\left(\frac{1}{e^{\delta \Delta_{t}}}-1\right) e^{-\delta(k-1) \Delta_{t}} \int_{0}^{(k-1) \Delta_{t}} e^{\delta s} d B_{s}^{(2)}
\end{gathered}
$$


since $\int_{0}^{(k-1) \Delta_{t}} e^{\delta s} d B_{s}^{(2)}$ is known when we know $V_{(k-1) \Delta_{t}}$ and $E\left[\int_{(k-1) \Delta_{t}}^{k \Delta_{t}} e^{\delta s} d B_{s}^{(2)} \mid V_{(k-1) \Delta_{t}}\right]=0$.

$$
\begin{aligned}
\operatorname{Var}\left[\ln V_{k \Delta_{t}} \mid V_{(k-1) \Delta_{t}}\right] & =\sigma^{2} \Delta_{t}+\mu_{2} \rho e^{-2 \delta k \Delta_{t}} E\left[\left(\int_{(k-1) \Delta_{t}}^{k \Delta_{t}} e^{\delta s} d B_{s}^{(2)}\right)^{2} \mid V_{(k-1) \Delta_{t}}\right] \\
& =\sigma^{2} \Delta_{t}+\mu_{2} \rho e^{-2 \delta k \Delta_{t}} \int_{(k-1) \Delta_{t}}^{k \Delta_{t}} e^{2 \delta s} d s \\
& =\sigma^{2} \Delta_{t}+\mu_{2} \rho e^{-2 \delta k \Delta_{t}} \frac{1}{2 \delta}\left(e^{2 \delta k \Delta_{t}}-e^{2 \delta(k-1) \Delta_{t}}\right) \\
& =\sigma^{2} \Delta_{t}+\frac{\mu_{2} \rho}{2 \delta}\left(1-e^{-2 \delta \Delta_{t}}\right)
\end{aligned}
$$

Hence, conditioned on the previous observation, $V_{k \Delta_{t}}$ follows log-normal distribution with above parameters. $V_{k \Delta_{t}}$ is a Markov process and we have

$$
L\left(V_{t}, t=0, \Delta_{t}, 2 \Delta_{t}, \ldots n \Delta_{t}\right)=f\left(V_{n \Delta_{t}} \mid V_{(n-1) \Delta_{t}}\right) f\left(V_{(n-1) \Delta_{t}} \mid V_{(n-2) \Delta_{t}}\right) \ldots . f\left(V_{\Delta_{t}} \mid V_{0}\right)
$$

where $f$ denotes density function.

Following Duan [5], we will also drop the first observation $V_{0}$ from the likelihood and use it to define the conditional distribution of the observations to follow; therefore, the above likelihood will not include the density of the first observation. Let

$$
\begin{gathered}
\operatorname{mean}_{k \Delta_{t}}=\left(\mu-\frac{1}{2} \sigma^{2}\right) \Delta_{t}-\sqrt{\frac{\mu_{2} \rho}{2 \delta}} Z_{0} e^{-\delta k \Delta_{t}}\left(1-e^{\delta \Delta_{t}}\right)-\sqrt{\mu_{2} \rho}\left(\frac{1}{e^{\delta \Delta_{t}}}-1\right) e^{-\delta(k-1) \Delta_{t}} \int_{0}^{(k-1) \Delta_{t}} e^{\delta s} d B_{s}^{(2)} \\
\operatorname{Var}_{k \Delta_{t}}=\operatorname{Var}\left[\ln V_{k \Delta_{t}} \mid V_{(k-1) \Delta_{t}}\right]
\end{gathered}
$$

Then, using density function of log-normal distribution,

$$
f\left(V_{k \Delta_{t}} \mid V_{(k-1) \Delta_{t}}\right)=\frac{1}{\sqrt{2 \pi \operatorname{Var}_{k \Delta_{t}}} V_{k \Delta_{t}}} e^{-\frac{\left(\ln \frac{V_{k \Delta_{t}}}{V_{(k-1) \Delta_{t}}}-\operatorname{mean}_{k \Delta_{t}}\right)^{2}}{2 \operatorname{Var}_{k \Delta_{t}}}}
$$

The log-likelihood function of unobserved $V_{k} \Delta_{t}$ becomes

$$
L\left(V_{t}, t=0, \Delta_{t}, 2 \Delta_{t}, \ldots n \Delta_{t}\right)=-\frac{n}{2} \ln 2 \pi-\frac{1}{2} \sum_{k=1}^{n} \ln \operatorname{Var}_{k \Delta_{t}}-\sum_{k=1}^{n} \frac{\left(\ln \frac{V_{k \Delta_{t}}}{V_{(k-1) \Delta_{t}}}-\operatorname{mean}_{k \Delta_{t}}\right)^{2}}{2 \operatorname{Var}_{k \Delta_{t}}}-\sum_{k=1}^{n} \ln V_{k \Delta_{t}}
$$

We have an element-by-element transformation from an unobserved asset sample to an observed equity sample through equation (8) and $\frac{\partial E_{t}}{\partial V_{t}}=N\left(d_{t}\right)$. Then, we can write log-likelihood function of equity as

$$
\left.L\left(E_{t}, t=0, \Delta_{t}, 2 \Delta_{t}, \ldots n \Delta_{t}\right) ; \mu, \delta, \mu_{2} \rho, Z_{0}, \sigma\right)=
$$




$$
-\frac{n}{2} \ln 2 \pi-\frac{1}{2} \sum_{k=1}^{n} \ln \operatorname{Var}_{k \Delta_{t}}-\sum_{k=1}^{n} \frac{\left(\ln \frac{\hat{V}_{k \Delta_{t}}}{\hat{V}_{(k-1) \Delta_{t}}}-\operatorname{mean}_{k \Delta_{t}}\right)^{2}}{2 \operatorname{Var}_{k \Delta_{t}}}-\sum_{k=1}^{n} \ln \hat{V}_{k \Delta_{t}}-\sum_{k=1}^{n} \ln N\left(\hat{d}_{k \Delta_{t}}\right)
$$

where $\hat{V}_{k \Delta_{t}}$ is the unique solution to (8) and $\hat{d}_{k \Delta_{t}}$ is $d_{k \Delta_{t}}$ with $V_{k \Delta_{t}}$ replaced by $\hat{V}_{k \Delta_{t}}$.

$\mu_{2} \rho$ will be estimated as one parameter, since we only use the product of these two parameters in the model.

\section{B Results}

Matlab R2015a was used for all the calculations and for producing graphs.

\section{B.1 Results (1)}
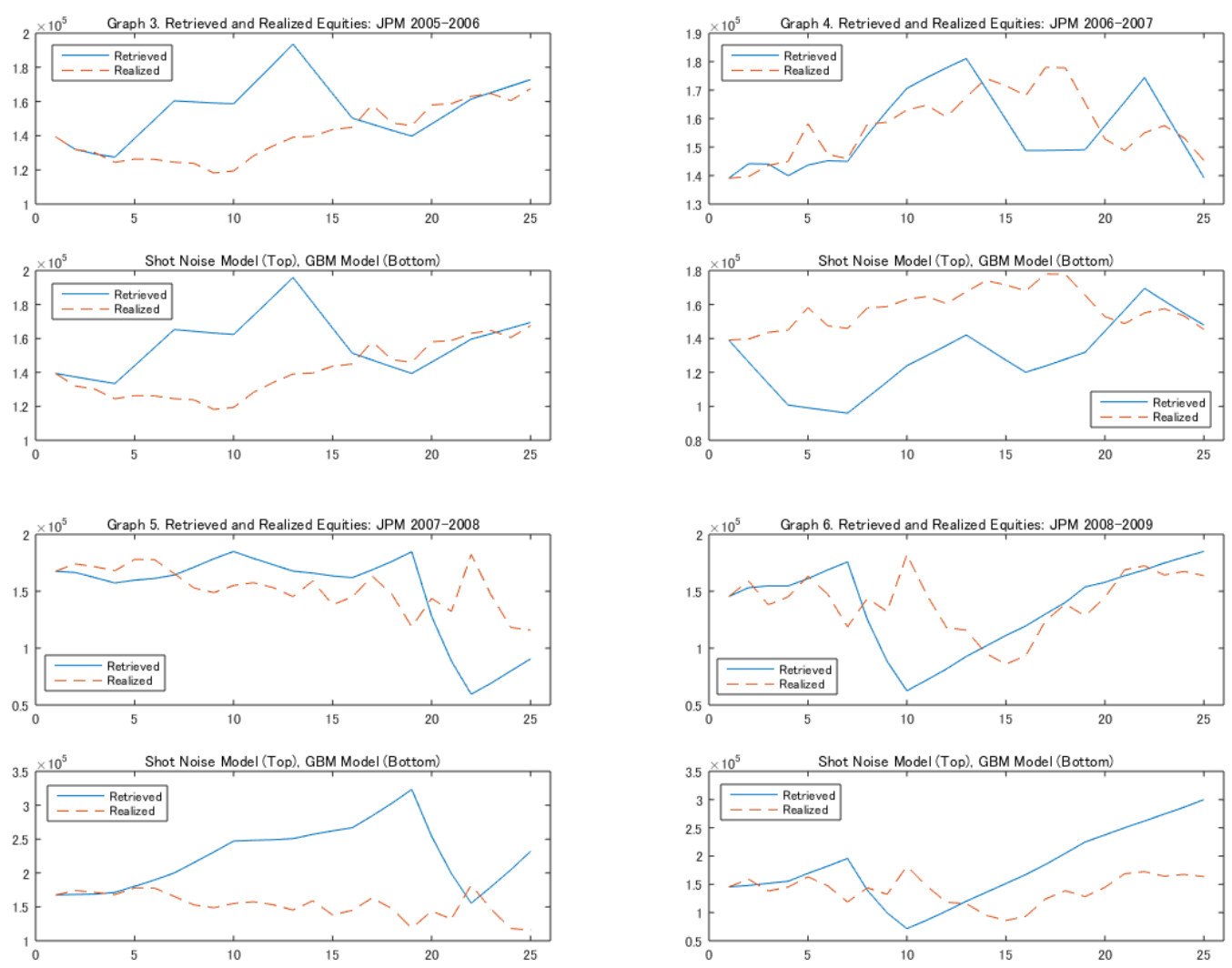

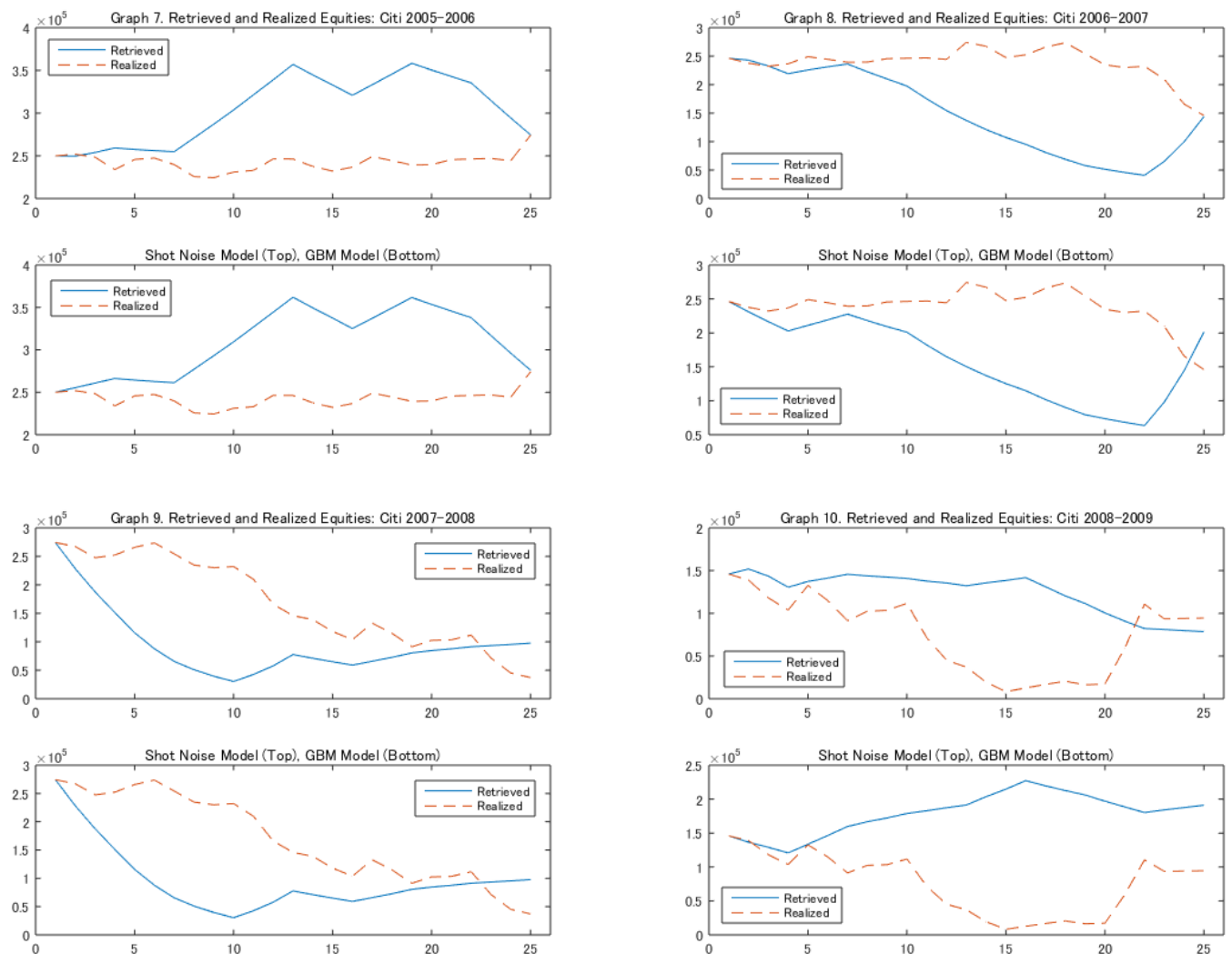

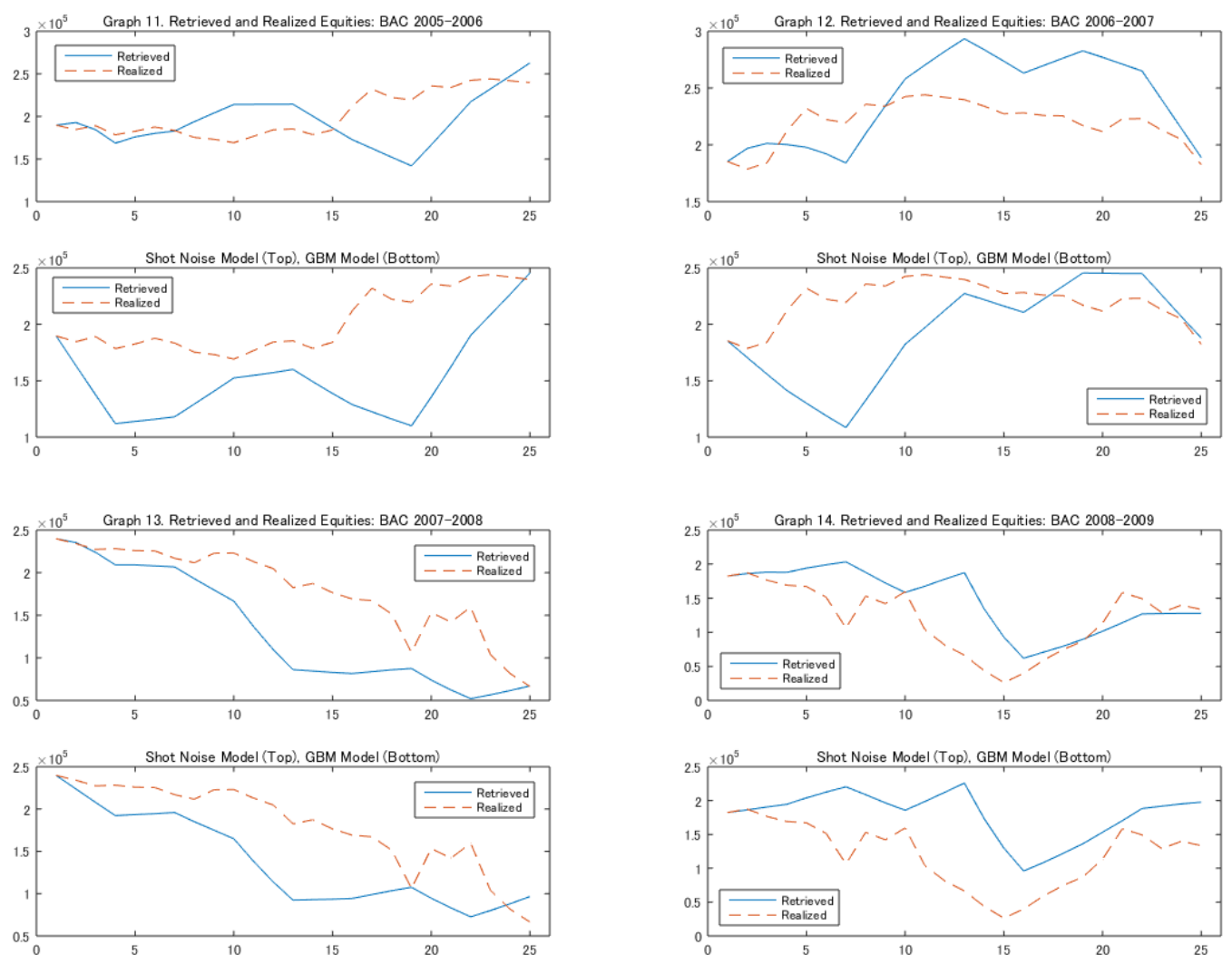


\section{B.2 Results (2)}
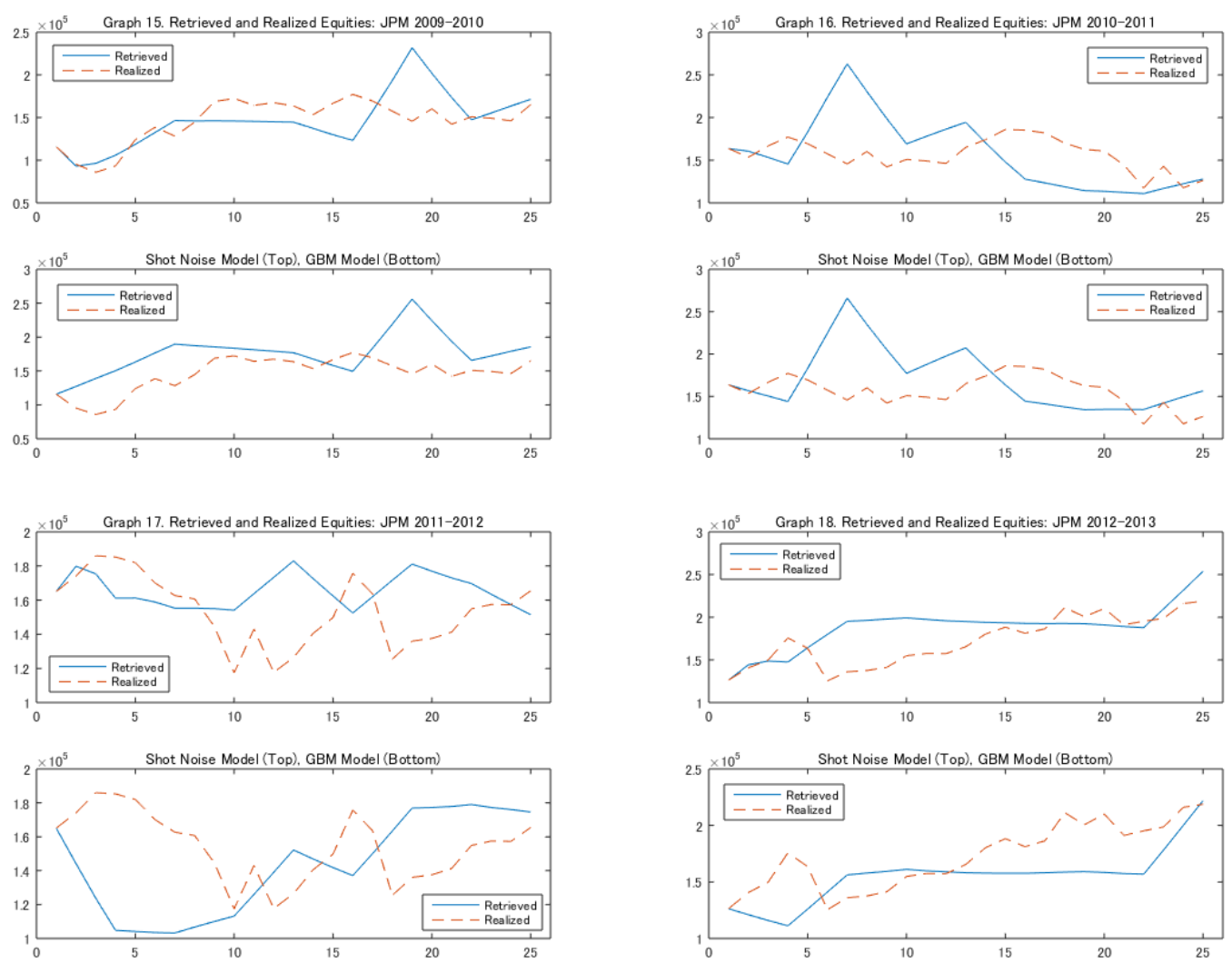

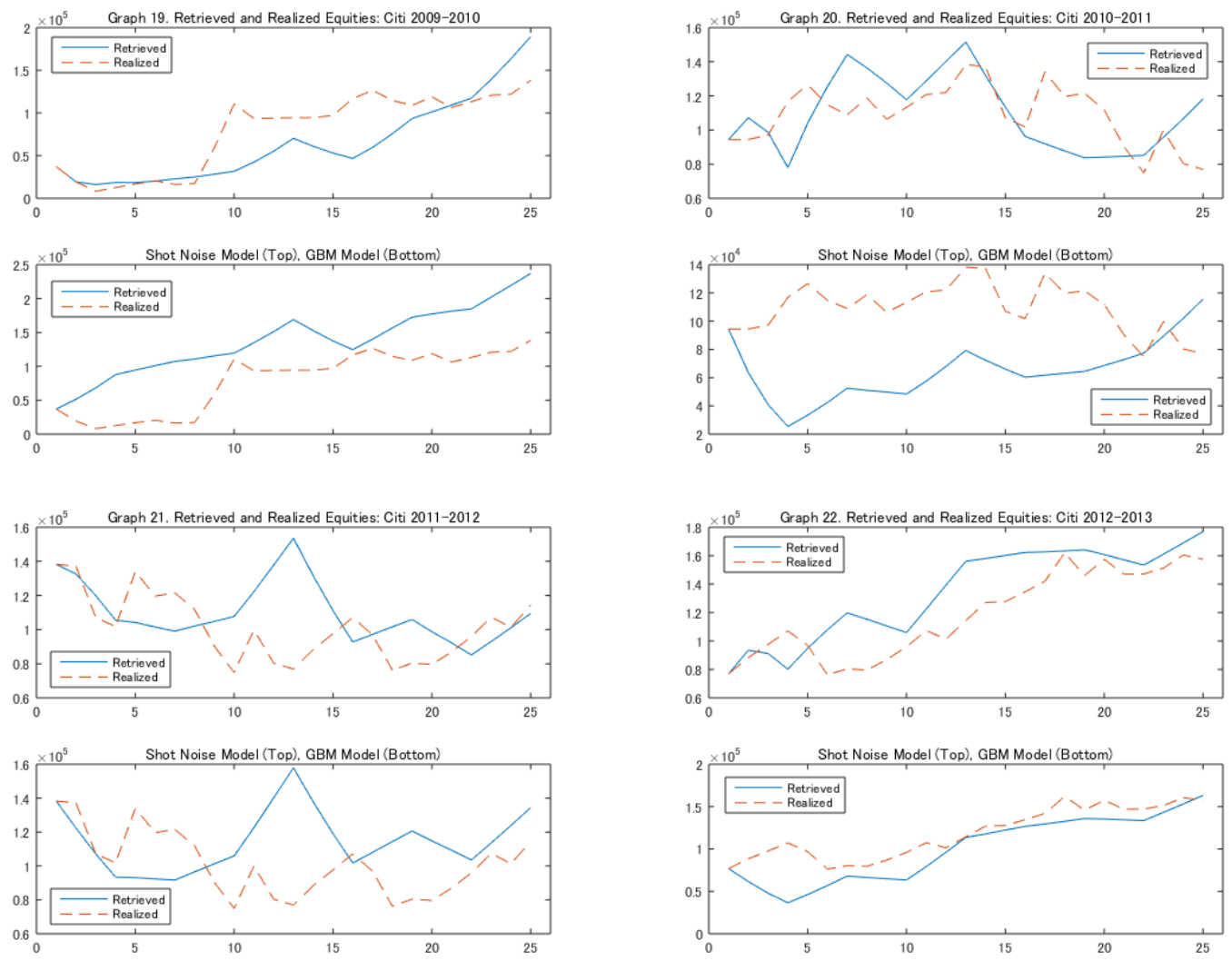

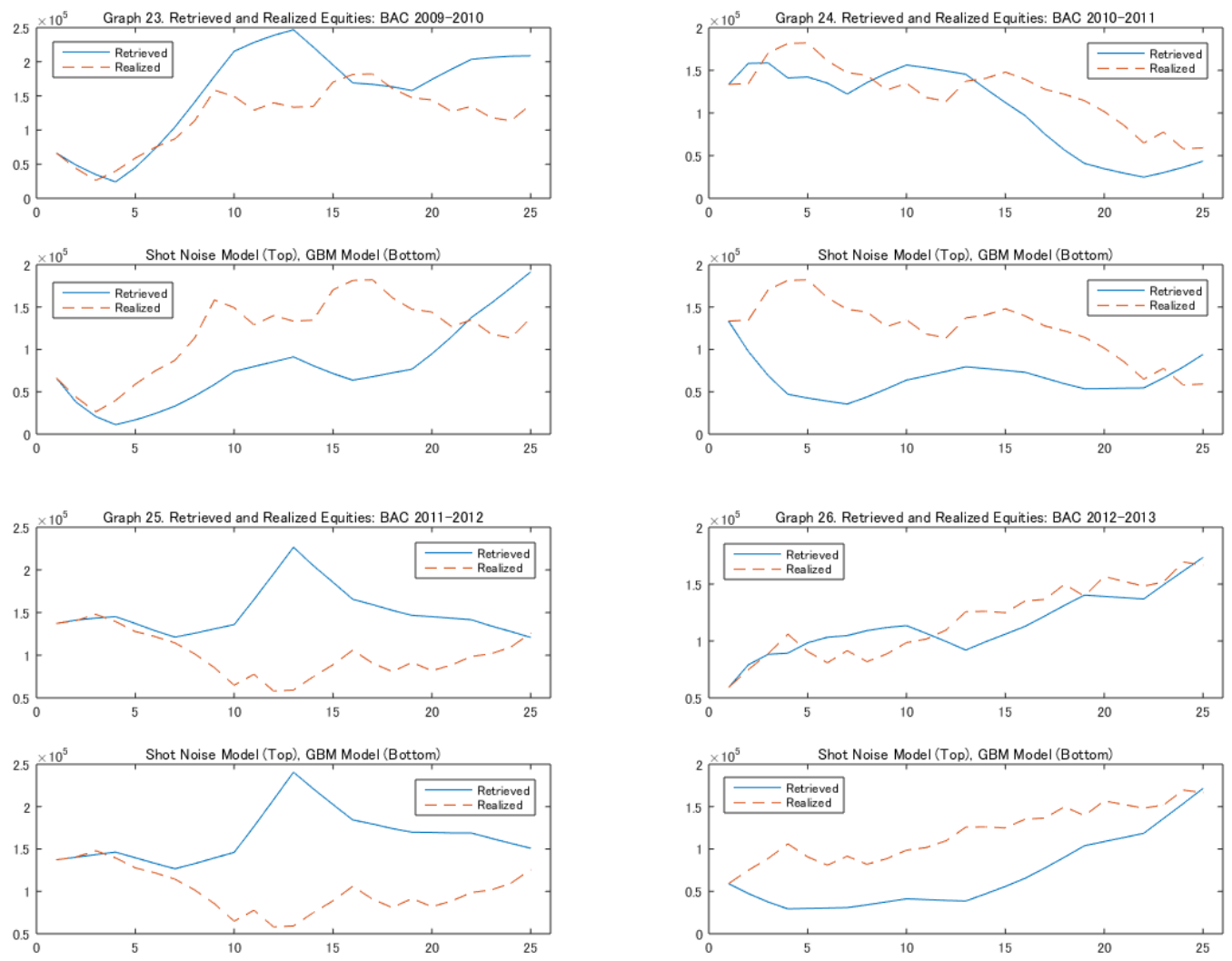
Table 15: 1-Yr Risk-free Interest Rates

\begin{tabular}{ccc}
\hline Simulation Year & Observed Date & 1-Yr Treasury Yield $(\%)$ \\
\hline 2007 & $12 / 29 / 06$ & 5 \\
2008 & $12 / 31 / 07$ & 3.34 \\
2009 & $12 / 31 / 08$ & 0.37 \\
2010 & $12 / 31 / 09$ & 0.47 \\
2011 & $12 / 31 / 10$ & 0.29 \\
2012 & $12 / 30 / 11$ & 0.12 \\
2013 & $12 / 31 / 12$ & 0.16 \\
2014 & $12 / 31 / 13$ & 0.13 \\
2015 & $12 / 31 / 14$ & 0.25
\end{tabular}

Source: U.S. Department of the Treasury 